\title{
On Optimal Sparse-Control Problems Governed by Jump-Diffusion Processes
}

\author{
Beatrice Gaviraghi'1, Andreas Schindele', Mario Annunziato², Alfio Borzì1 \\ ${ }^{1}$ Institut für Mathematik, Universität Würzburg, Würzburg, Germany \\ ${ }^{2}$ Dipartimento di Matematica, Università degli Studi di Salerno, Fisciano (SA), Italy \\ Email: beatrice.gaviraghi@mathematik.uni-wuerzburg.de, andreas.schindele@posteo.de, mannunzi@unisa.it, \\ alfio.borzi@mathematik.uni-wuerzburg.de
}

How to cite this paper: Gaviraghi, B., Schindele, A., Annunziato, M. and Borzì, A. (2016) On Optimal Sparse-Control Problems Governed by Jump-Diffusion Processes. Applied Mathematics, 7, 1978-2004. http://dx.doi.org/10.4236/am.2016.716162

Received: July 25, 2016

Accepted: October 22, 2016

Published: October 25, 2016

Copyright $\odot 2016$ by authors and Scientific Research Publishing Inc. This work is licensed under the Creative Commons Attribution International License (CC BY 4.0).

http://creativecommons.org/licenses/by/4.0/

\begin{abstract}
A framework for the optimal sparse-control of the probability density function of a jump-diffusion process is presented. This framework is based on the partial integro-differential Fokker-Planck (FP) equation that governs the time evolution of the probability density function of this process. In the stochastic process and, correspondingly, in the FP model the control function enters as a time-dependent coefficient. The objectives of the control are to minimize a discrete-in-time, resp. continuous-intime, tracking functionals and its $L^{2}$ - and $L^{1}$-costs, where the latter is considered to promote control sparsity. An efficient proximal scheme for solving these optimal control problems is considered. Results of numerical experiments are presented to validate the theoretical results and the computational effectiveness of the proposed control framework.
\end{abstract}

\section{Keywords}

Jump-Diffusion Processes, Partial Integro-Differential Fokker-Planck Equation, Optimal Control Theory, Nonsmooth Optimization, Proximal Methods

\section{Introduction}

Recently, largely motivated by computational finance applications, there has been a growing interest in stochastic jump-diffusion processes. In fact, empirical facts suggest that a discontinuous path could be most appropriate for describing the dynamics of stock prices; see [1] and references therein. Therefore, in many application models, the stock price is modeled by a jump-diffusion stochastic process, rather than by an Itô-diffusion process [2] [3]. In this framework, when option pricing models and portfolio optimization problems are considered, partial integro-differential equations 
(PIDEs) naturally arise; see [1] [4] and references therein. In the present paper, we focus on a stochastic jump-diffusion (JD) process, whose jump component is given by a compound Poisson process subject to given barriers. Also concerning market models, systems driven by Poisson processes have been considered; see, e.g., [5].

When one considers decision making issues involving random quantities, stochastic optimization problems must be solved. Such problems have largely been examined in the scientific literature, because of the numerous applications in, e.g., physics, biology, finance, and economy [6]-[8]. In these references, the usual procedure consists of minimizing a deterministic objective function that depends on the state and on the control variables. However, within this approach, statistical expectation objectives must be considered, since the state evolution is subject to randomness.

In this work, we tackle the issue of controlling a stochastic process by following an alternative approach already proposed in [9]-[11], where the problem is reformulated from stochastic to deterministic. The key idea of this strategy is to focus on the probability density function (PDF) of the considered process, whose time evolution is modeled by the Fokker-Planck (FP) equation, also known as the Kolmogorov forward equation. The FP control approach is advantageous since it allows to model the action of the control over the entire space-time range of the underlying process, which is characterized by the shape of its PDF.

In the case of our JD process, the FP equation takes the form of a PIDE endowed with initial and boundary conditions. While the Cauchy data must be the initial distribution of the given random variable, the boundary conditions of a FP problem depend on the considered model. For the derivation of the FP equation and a discussion about boundary conditions, see [12]-[14]. Starting from the controlled stochastic differential model, the coefficients of the FP equation and thus the control mechanism are authomatically determined and thus an infinite dimensional optimal control problem governed by the FP PIDE related to a JD process is obtained. Since the control variable enters the state equation as a coefficient of the partial integro-differential operator, the resulting optimization problem is nonconvex.

Infinite-dimensional optimization is a very active research field, motivated by a broad range of applications ranging from, e.g., fluid flow, space technology, heat phenomena, and image reconstruction; see, e.g., [15]-[17]. The main focus of this research work has been on problems with smooth cost functionals governed by partial differential equations (PDEs) with linear control mechanism [16] [17]. However, bilinear control problems governed by parabolic and elliptic PDEs have been also recently investigated; see, e.g., [9] [18] [19] and references therin. In these references, the purpose is often to compute optimal controls such that an appropriate norm of the difference between a given target and the resulting state is minimized. In the present paper, we consider tracking objectives that include mean expectation values as in [20]. Our framework aims at the minimization of the difference between a known sequence of values and the first moment of a JD process, such that our formulation can also be considered as a parameter estimation problem for stochastic processes. In the discrete-in-time case, 
the form of the cost functional gives rise to a finite number of discontinuities in time in the adjoint variable and hence of the control. A similar situation has already been considered in [21].

Very recently, PDE-based optimal control problems with sparsity promoting $L^{1}$-cost functionals have been investigated starting with [22]. See [19] for a short survey and further references. Such formulation gives rise to a sparse optimal control, and for their solution variants of the semismooth Newton (SSN) method [23] have been considered. An alternative to such techniques is represented by proximal iterative schemes, introduced in [24] and [25] and further developed in the framework of finite-dimensional optimization [26] [27]. Recents works have adapted the structure of these algorithms for solving infinite-dimensional PDE optimization problems [19]. Moreover, it has been shown in [19] that in infinite-dimensional problems, proximal algorithms have a computational performance comparable to SSN methods while they do not require the construction of the second-order derivatives. In the present paper, we consider a $L^{1}$ cost functional and apply the proximal algorithm proposed in [19] [28]. One of the novelties of our work consists of combining pioneering techniques for nonsmooth problems with the control of the PDF of a FP PIDE of a JD process.

This paper is organized as follows. In the next section, we discuss the functional setting of the FP problem modeling the evolution of the PDF of a JD stochastic process. In Section 3, we formulate our optimal control problems. Section 4 is devoted to the formulation of the corresponding first-order optimality systems. In Section 5, we discuss the discretization of the state and adjoint equations of the optimality system. In Section 6 , we illustrate a proximal method for solving our optimal control problems. Section 7 is devoted to presenting results of numerical tests, including a discussion on the robustness of the algorithm to the choice of the parameters of the optimization problem. A section of conclusions completes this work.

\section{The Fokker-Planck Equation of a Jump-Diffusion Process}

In this section, we introduce a JD process and the corresponding FP equation that models the time evolution of the PDF of this process. Further, we discuss well-posedness and regularity of solutions to our FP problem.

We consider a time interval $I:=\left[t_{0}, t_{f}\right]$ and a stochastic process $\left\{X_{t}\right\}_{t \in I}$ with range in a bounded domain $\Omega \subset \mathbb{R}^{n}$. We assume that the set $\Omega$ is convex with Lipschitz boundary. The dynamic of $\mathrm{X}$ is governed by the following initial value problem

$$
\left\{\begin{array}{l}
\mathrm{d} X_{t}=b\left(X_{t}, t\right) \mathrm{d} t+\sigma\left(X_{t}\right) \mathrm{d} W_{t}+\mathrm{d} P_{t} \\
X_{t_{0}}=X_{0},
\end{array}\right.
$$

where $X_{0} \in \mathbb{R}^{n}$ is a random variable with known distribution. The functions $b: \Omega \times I \rightarrow \mathbb{R}^{n}$ and $\sigma: \Omega \rightarrow \mathbb{R}^{n \times d}$ represent the drift and the diffusion coefficients, respectively. We assume that $\sigma$ is full rank. Random increments to the process are given by a Wiener process $W \in \mathbb{R}^{d}$ and a compound Poisson process $P \in \mathbb{R}^{n}$. The rate of jumps and the jump distribution are denoted with $\lambda \in \mathbb{R}^{+}$and $\hat{g}$, respectively. 
Define $a: \Omega \rightarrow \mathbb{R}^{n \times n}, a_{i j}(x):=\sum_{k=1}^{d} \frac{1}{2} \sigma_{i k}(x) \sigma_{j k}(x)$. Since $\sigma$ is full rank, $a$ is positive definite, and hence there exists $c_{a}>0$ such that

$$
v^{\mathrm{T}} a(x) v \geq c_{a}\|v\|_{\mathbb{R}^{n}}^{2}, \quad \text { for each } v \in \mathbb{R}^{n} \text {, a.e. in } \Omega .
$$

In this work, we consider a stochastic process with reflecting barriers. This assumption determines the boundary conditions for the FP equation corresponding to (1), see below. Define $Q:=\Omega \times I$ and $\Sigma:=\partial \Omega \times I$, and denote with $f$ the PDF of the process given by (1). It is known [12] [13] that the time evolution of $f$ is modeled by the following FP of PIDE type

$$
\partial_{t} f(x, t)=\mathcal{L} f(x, t)+\mathcal{I f}(x, t), \quad(x, t) \in Q,
$$

where the differential operator $\mathcal{L}$ and the integral operator $\mathcal{I}$ are defined as follows

$$
\mathcal{L} f(x, t):=-\sum_{i=1}^{n} \partial_{i}(b(x, t) f(x, t))+\sum_{i, j=1}^{n} \partial_{i j}^{2}(a(x) f(x, t)),
$$

and

$$
\text { If }(x, t):=\lambda\left[\int_{\mathbb{R}^{n}} f(y, t) g(x, y) \mathrm{d} y-f(x, t)\right],
$$

respectively. The definition of $g$ in (5) takes into account the presence of reflecting barriers and the dependence on the jump amplitude $\hat{g}$, as we discuss later.

Notice that the differential operator $\mathcal{L}$ can be rewritten as follows

$$
\mathcal{L} f(x, t):=\nabla \cdot F(x, t),
$$

where

$$
F(x, t):=B(x, t) f(x, t)+a(x) \nabla f(x, t) .
$$

and

$$
B_{i}(x):=\sum_{j=1}^{n} \partial_{j} a_{i j}(x)-b_{i}(x, t),
$$

for each $i=1, \cdots, n$. The function $F$ in (6) represents the flux of the differential operator $L$, and $-F$ is known in the literature as the probability current in case of stochastic processes without jumps [13].

The PDF $f$ of $X$ in (1) in the bounded domain $\Omega$ is obtained by solving (3), endowed by suitable initial and boundary conditions. In our setting, the initial data $f_{0}$ represents the PDF of the initial random variable $X_{0}$. The choice of a bounded domain with reflecting barriers results in the following zero-flux boundary conditions for the FP model

$$
F(x, t) \cdot \hat{n}(x, t)=0 \text { for }(x, t) \in \Sigma,
$$

where $\hat{n}$ is the unit outward normal on $\partial \Omega$.

Notice that the flux $F$ corresponds to the differential part of the FP equation, that is, to the drift and diffusion components of the stochastic process. In order to take into account the action of a reflecting barrier on the jumps, we consider a suitable definition 
of the kernel $g$, which can be conveniently illustrated in the one-dimensional case as follows.

Consider $n=1$ and $\Omega=(r, s)$. The kernel $g$ in (5) takes the following form

$$
\begin{aligned}
g(x, y) & :=\hat{g}(x-y)+\hat{g}(2 r-x-y) H(r+s-x-y) \\
& +\hat{g}(2 s-x-y) H(x+y-r-s),
\end{aligned}
$$

where $H$ is the Heaviside step function defined by

$$
H(z):=\left\{\begin{array}{cc}
1, & z>0 \\
0, & z<0 \\
1 / 2, & z=0
\end{array}\right.
$$

We normalize $g$ and $\hat{g}$ such that

$$
\int_{r-s}^{s-r} \hat{g}(z) \mathrm{d} z=\int_{\mathbb{R}} g(x, y) \mathrm{d} x=1 \text { for all } y \in \Omega .
$$

The next remark motivates the choice of the boundary conditions (8) and of the condition (10).

Remark 2.1. Assume (8) and (10). Provided that $f_{0}$ is a PDF in $\Omega$, then the solution to our FP problem satisfies the following conservation equation

$$
\frac{\mathrm{d}}{\mathrm{d} t} \int_{\Omega} f(x, t) \mathrm{d} x=0 \text { for each } t \in\left(t_{0}, t_{f}\right] .
$$

That is, the total probability over the space domain $\Omega$ at each time $t \in I$ is preserved, in the sense that

$$
\int_{\Omega} f(x, t) \mathrm{d} x=\int_{\Omega} f_{0}(x) \mathrm{d} x \text { for each } t \in\left(t_{0}, t_{f}\right] .
$$

Our FP problem is stated as follows

$$
\begin{cases}\partial_{t} f(x, t)=\nabla \cdot F(x, t)+\mathcal{I} f(x, t) & \text { for }(x, t) \in Q \\ f(x, 0)=f_{0}(x) & \text { for } x \in \Omega \\ F(x, t) \cdot n(x, t)=0 & \text { for }(x, t) \in \Sigma .\end{cases}
$$

Next, we recall some definitions concerning the functional spaces needed to state the existence and uniqueness of solutions to (11). The space $C^{0}(\Omega)$ refers to the functions that are continuous in $\Omega$ and it is endowed with the supremum norm. Let $\alpha$ be a constant, $\alpha>1 / 2$. The space $C^{\alpha}(\Sigma)$ refers to the functions that are Hölder continuous on $\Sigma$, with Hölder exponent $\alpha$ with respect to the space variable. The space $L^{\infty}(\Omega)$ denotes all the functions that are bounded on $\Omega$, up to a set of zero measure. The spaces $H^{1}(\Omega)$ and $H^{2,1}(Q)$ are defined as follows

$$
\begin{aligned}
& H^{1}(\Omega):=\left\{v \in L^{2}(\Omega) \mid \partial_{i} v \in L^{2}(\Omega), i=1, \cdots, n\right\}, \\
& H^{2,1}(Q):=\left\{v \in L^{2}(Q) \mid \partial_{t} v, \partial_{i} v, \partial_{i j}^{2} v \in L^{2}(Q), i, j=1, \cdots, n\right\} .
\end{aligned}
$$

These spaces are endowed with the following norms

$$
\|v\|_{H^{1}(\Omega)}:=\sum_{|i|=0}^{1}\left\|\partial^{i} v\right\|_{L^{2}(\Omega)}, \quad\|v\|_{H^{2,1}(Q)}:=\sum_{2 j+i \mid=0}^{2}\left\|\partial_{t}^{j} \partial_{x}^{i} v\right\|_{L^{2}(Q)},
$$

where $i, j \in \mathbb{N}^{n}$ denote multi-indeces. 
We assume that the coefficients $a$ and $b$ in (4) satisfy the following conditions

$$
\begin{aligned}
& a(x) \in C^{0}(\Omega) \cap C^{\alpha}(\partial \Omega), \partial_{i} a(x) \in L^{\infty}(\Omega) \cap C^{\alpha}(\partial \Omega), \partial_{i j}^{2} a(x) \in L^{\infty}(\Omega) \\
& b_{i}(x, t) \in L^{\infty}(Q) \cap C^{\alpha}(\Sigma), \partial_{i} b_{j}(x, t) \in L^{\infty}(Q)
\end{aligned}
$$

for each $i, j=1, \cdots, n$.

Notice that $a$ and $b$ must be defined on the closure $\bar{\Omega}$ due to their role in the boundary conditions in (11). We assume that the following condition is satisfied

$$
\int_{\mathbb{R}^{n}} \frac{\|y\|^{2}}{(1+\|y\|)} g(y) \mathrm{d} y=C_{0}<\infty,
$$

with $C_{0}>0$ and $\|y\|:=\left(\sum_{j=1}^{n} y_{j}^{2}\right)^{1 / 2}$ for $y \in \mathbb{R}^{n}$.

We have the following theorem [29].

Theorem 2.1. Assume $\partial \Omega \in C^{2}$. Let the coefficients $a$ and $b$ in $L$ in (4) and that $g$ satisfy the assumptions (13) and (14), respectively. Then, for given $f_{0} \in H^{1}(\Omega)$, the initial-boundary value problem (11) admits a unique solution $f \in H^{2,1}(Q)$.

Proof. See [29].

Remark 2.2. Provided that $f_{0}$ is also a PDF, it follows by standard arguments [29] that $f(x, t) \geq 0$ for each $(x, t) \in Q$.

Consider the following spaces $V:=H^{1}(\Omega), H:=L^{2}(\Omega)$. We denote with $V^{*}$ the dual space of $V$ and with $\langle\cdot, \cdot\rangle_{V^{*} V}$ their canonical pairing. We consider the following Gelfand triple $V \subset H \subset V^{*}$, that exploits the natural isomorphism $H \simeq H^{*}$ between a Hilbert space with his dual. Each embedding is dense and continuous [17].

Given the interval $I=\left[t_{0}, t_{f}\right]$ and an arbitrary Banach space $Z$, we define the following spaces

$$
\begin{aligned}
& L^{2}(I ; Z)=\left\{y: I \rightarrow Z \text { such that } \int_{I}\|y(t)\|_{Z}^{2} \mathrm{~d} t<\infty\right\} \\
& C(I ; Z)=\left\{y: I \rightarrow Z \text { such that } \lim _{\tau \rightarrow t}\|y(\tau)-y(t)\|_{Z}=0 \quad \forall t \in I\right\},
\end{aligned}
$$

which are also Banach spaces [17] equipped with the following norms

$$
\|y\|_{L^{2}(I ; Z)}:=\left(\int_{I}\|y(t)\|_{Z}^{2} \mathrm{~d} t\right)^{\frac{1}{2}} \text { and }\|y\|_{C(I ; Z)}:=\max _{t \in I}\|y(t)\|_{Z},
$$

respectively. We consider the following space

$$
W:=\left\{y \in L^{2}(I ; V) \text { with } \partial_{t} y \in L^{2}\left(I ; V^{*}\right)\right\},
$$

which is a Hilbert space [17] with respect to the scalar product defined as follows

$$
(f, g)_{W}:=\int_{I}(f, g)_{V}+\int_{I}\left(\partial_{t} f, \partial_{t} g\right)_{V^{*}} .
$$

With this preparation, we can recall the following theorem [17].

Theorem 2.2. The embedding $W \hookrightarrow C(I ; H)$ is continuous. Therefore, every $y \in W$ coincides with an element of $C(I ; H)$, up to a set of null measure.

The following proposition provides a useful a priori estimate of the solution to (11).

Proposition 2.3. Let $f_{0} \in H^{1}(\Omega), \quad f_{0} \geq 0$, and $g$ satisfies (14). Then if $f$ is a solution 
to (11), the following inequality holds

$$
\|f\|_{L^{2}(I ; V)}+\|f\|_{L^{\infty}(I ; H)}+\left\|\partial_{t} f\right\|_{L^{2}\left(I ; V^{*}\right)} \leq C\left\|f_{0}\right\|_{L^{2}(\Omega)} .
$$

Proof. Consider the $H$ inner product of the equation in (11) with $f$. Exploiting the properties of the Gelf and triple, we have

$$
\left\langle\partial_{t} f, f\right\rangle_{V^{*} V}=\int_{\Omega}(\nabla \cdot F) f+\int_{\Omega} \mathcal{I}(f) f .
$$

We make use of the following fact [17], $\left\langle\partial_{t} f, f\right\rangle_{V^{*} V}=\frac{1}{2} \frac{\mathrm{d}}{\mathrm{d} t}\|f(t)\|_{L^{2}(\Omega)}^{2}$. The terms on the right-hand side in (19) are recast as follows.

First, we exploit the zero-flux boundary conditions in (11) and the coercivity of a as given in (2). Moreover, we make use of the following Cauchy inequality

$$
\alpha \beta \leq \frac{\alpha^{2}}{2 \varepsilon^{2}}+\frac{\beta^{2} \varepsilon^{2}}{2},
$$

which holds for each $\alpha, \beta \in \mathbb{R}$ and $\varepsilon>0$. Integrating by parts and recalling the definition of $F$ in (6), we have

$$
\begin{aligned}
\int_{\Omega}(\nabla \cdot F) f & =\int_{\partial \Omega} f F \cdot \partial v-\int_{\Omega} F \cdot \nabla f=\int_{\Omega}-f B \cdot \nabla f-\int_{\Omega} \nabla f^{\mathrm{T}} a \nabla f \\
& \leq \int_{\Omega} \frac{B^{\mathrm{T}} B f^{2}}{2 \varepsilon^{2}}+\int_{\Omega} \frac{\nabla f^{\mathrm{T}} \nabla f \varepsilon^{2}}{2}-c_{a}\|\nabla f\|_{L^{2}(\Omega)}^{2} .
\end{aligned}
$$

We choose $\varepsilon:=\sqrt{2 c_{a}}$, where $c_{a}$ is defined in (2), and define

$$
c_{B}:=\sum_{i=1}^{n}\left\|B_{i}\right\|_{\infty}^{2}=\sum_{i=1}^{n}\left\|\sum_{j=1}^{n} \partial_{j} a_{i j}(x)-b_{i}(x, t)\right\|_{\infty}^{2} .
$$

We have $C_{B}<\infty$ thanks to (13).

Therefore we have

$$
\int_{\Omega}(\nabla \cdot F) f \leq \frac{C_{B}}{4 c_{a}}\|f(t)\|_{L^{2}(\Omega)}^{2} .
$$

Recalling the definition of $I$ in (5) and defining $c_{G}:=\|g\|_{\infty}$, we have

$$
\int_{\Omega} \mathcal{I}(f) f \leq \lambda c_{G}\|f(t)\|_{L^{2}(\Omega)}^{2}-\lambda\|f(t)\|_{L^{2}(\Omega)}^{2} .
$$

Since $\Omega$ is bounded, we have

$$
\|f(t)\|_{L^{1}(\Omega)} \leq \sqrt{|\Omega|}\|f(t)\|_{L^{2}(\Omega)} .
$$

Therefore,

$$
\int_{\Omega} \mathcal{I}(f) f \leq \lambda|\Omega|\left|c_{G}-1\right|\|f(t)\|_{L^{2}(\Omega)}^{2}
$$

Define $c:=\frac{c_{B}}{2 c_{a}}+2 \lambda|\Omega|\left|c_{G}-1\right|$. Note that $c$ is a bounded time-dependent function. The estimates in (20) and (21) allow us to write (19) as follows

$$
\frac{\mathrm{d}}{\mathrm{d} t}\|f(t)\|_{L^{2}(\Omega)}^{2} \leq c\|f(t)\|_{L^{2}(\Omega)}^{2}
$$

By applying the Gronwall inequality, we have 


$$
\|f(t)\|_{L^{2}(\Omega)} \leq\left\|f_{0}\right\|_{L^{2}(\Omega)} \exp \left\{\frac{1}{2} \int_{t_{0}}^{t} c \mathrm{~d} s\right\}
$$

Next, we outline how to obtain an upper bound of $\|\nabla f(t)\|_{L^{2}(\Omega)}$. We integrate (2) over $\Omega$ and then recall the definition of $F$ in (6). We have

$$
\begin{aligned}
c_{a}\|\nabla f(t)\|_{L^{2}(\Omega)}^{2} & \leq \int_{\Omega}(\nabla f)^{\mathrm{T}} a \nabla f=\int_{\Omega}(\nabla f)^{\mathrm{T}}(F-B f) \\
& \leq \int_{\partial \Omega} f F \cdot \partial n-\int_{\Omega} f(\nabla \cdot F)-\int_{\Omega} f(\nabla f)^{\mathrm{T}} B \\
& \leq \int_{\Omega} f\left(\partial_{t} f-\mathcal{I}(f)\right)-\int_{\Omega} f(\nabla f)^{\mathrm{T}} B,
\end{aligned}
$$

where we used the PIDE and the boundary condition of the FP problem in (11). Proceeding as above, we obtain

$$
\|\nabla f(t)\|_{L^{2}(\Omega)} \leq \bar{C}\left\|f_{0}\right\|_{L^{2}(\Omega)},
$$

with $\bar{C}>0$. This last estimate, together with (22), proves that

$$
\|f\|_{L^{2}(I ; V)} \leq C\left\|f_{0}\right\|_{L^{2}(\Omega)},
$$

up to a redefinition of the constant $c>0$. The estimates of the other addends in (18) follow after some calculation with arguments as in [9] [17].

Proposition 2.4. Assume (13) and $f_{0} \in L^{2}(\Omega)$. Then the unique solution to (11) belongs to $L^{2}(I ; V) \cap L^{\infty}(I ; V)$, with $\partial_{t} f \in L^{2}\left(I ; V^{*}\right)$. Moreover, $f \in C(I ; H)$.

Proof. The statement follows from the a priori estimates of Proposition 2.3 and Theorem 2.2.

We define $F:=\left\{f \in W \mid f(0)=f_{0}\right\}$, where $f_{0}$ is the initial data in (11). The initial-boundary value problem (11) can be stated as $\mathcal{K}(f)=0$, where the map $\mathcal{K}$ is defined as follows

$$
\kappa(f):= \begin{cases}\partial_{t} f-\nabla \cdot F(f)-\mathcal{I} f & \text { in } Q \\ F(f), & \text { on } \Sigma,\end{cases}
$$

with $F$ and $I$ defined in (6) and (5), respectively.

\section{Two Fokker-Planck Optimal Control Problems}

In this section, we define our optimal control problems governed by (23) and prove the existence of at least an optimal solution.

We consider a control mechanism that acts on the drift function $b=b(x ; u)=b(x, t)$ by means of a time-dependent control $u=u(t) \in \mathbb{R}$. Therefore we refer to (23) as $\mathcal{K}(f, u)=0$. We assume that $b$ is a smooth function of its arguments and that assumption (13) is fulfilled. We remark that a time-dependent control function is a natural choice considering that it originates from the stochastic differential model where the time is the only independent variable.

We assume the presence of control constraints given by $u_{a}, u_{b} \in \mathbb{R}$, with $u_{a}<0<u_{b}$. We denote

$$
\mathcal{U}:=L^{2}(I)
$$




$$
\mathcal{U}_{a d}:=\left\{u \in \mathcal{U}: u_{a} \leq u \leq u_{b}\right\} \subset \mathcal{U} .
$$

Remark 3.1. The subset $\mathcal{U}_{a d}$ is nonempty, closed, and convex.

Let $v$ and $\gamma$ be positive constants. We consider the following objective

$$
\mathcal{J}(f, u):=D(f)+\frac{v}{2}\|u\|_{2}^{2}+\gamma\|u\|_{1} .
$$

The term $D(f)$ in (26) represents a tracking objective that involves the expectation value of $X_{t}, \mathbb{E}\left[X_{t}\right]:=\int_{\Omega} x f(x, t) \mathrm{d} x$, and a desired trajectory or a discrete set of values (e.g. measurements). We investigate the following two cases.

1) Given a set of values $\left\{\xi_{k}\right\}_{k=1}^{K}$ at different times $t_{k} \in\left(t_{0}, t_{f}\right), \forall k=1, \cdots, K$, we have

$$
D(f):=\frac{1}{K} \sum_{k=1}^{K}\left(\xi_{k}-\int_{\Omega} x f\left(x, t_{k}\right) \mathrm{d} x\right)^{2} .
$$

2) Given a square-integrable function $\xi: I \rightarrow \mathbb{R}$, we have

$$
D(f):=\int_{t_{0}}^{t_{f}}\left(\xi(t)-\int_{\Omega} x f(x, t) \mathrm{d} x\right)^{2} \mathrm{~d} t .
$$

The norms in (26) are defined as follows

$$
\|u\|_{2}:=\left(\int_{I}|u(t)|^{2} \mathrm{~d} t\right)^{1 / 2} \text { and }\|u\|_{1}:=\int_{I}|u(t)| \mathrm{d} t .
$$

Remark 3.2. The choice of a bounded time interval I ensures that the $L^{1}$-norm is finite whenever $u \in U$.

Remark 3.3. The functional $\mathcal{J}$ is convex and continuous with respect to $(f, u)$ in the $L^{2}$ norm.

We investigate the following optimal control problem (s)

$$
\begin{aligned}
& \min \mathcal{J}(f, u) \\
& \text { such that } \mathcal{K}(f, u)=0, \quad(f, u) \in \mathcal{F} \times \mathcal{U}_{a d} .
\end{aligned}
$$

In order to discuss the existence and uniqueness of solutions to (29), we consider the control-to-state operator $\mathcal{S}: \mathcal{U} \rightarrow \mathcal{F}$, that maps a given $u \in \mathcal{U}$ into $\mathcal{S}(u):=f \in \mathcal{F}$, where $(f, u)$ satisfies $\mathcal{K}(f, u)=0$. Note that the definition of $\mathcal{U}_{a d}$ in (25) ensures that b satisfies (13). Because of Theorem 2.1, the operator $\mathcal{S}$ is well defined.

The next proposition can be proved by using standard arguments [9] [17].

Proposition 3.1. The mapping $\mathcal{S}: \mathcal{U} \rightarrow C(I ; H), u \mapsto f=\mathcal{S}(u)$ solution to (11) is Fréchet differentiable and the directional derivative $e:=\mathcal{S}^{\prime}(u) \cdot h$ satisfies the following initial-boundary problem

$$
\begin{cases}\partial_{t} e=\nabla \cdot F(e)+\mathcal{I}(e)-\nabla \cdot\left(f b^{\prime}(u) h\right) & \text { for }(x, t) \in Q, \\ {\left[F(e)-f b^{\prime}(u) h\right] \cdot n=0} & \text { for }(x, t) \in \Sigma, \\ e\left(x, t_{0}\right)=0 & \text { for } x \in \Omega,\end{cases}
$$

where $b$ is the drift in (1) and $F$ is defined in (6).

The constrained optimization problem (29) can be transformed into an unconstrained one as follows 


$$
\min _{u \in \mathcal{U}_{a d}} \hat{\mathcal{J}}(u)
$$

where $\hat{\mathcal{J}}: u \mapsto \hat{\mathcal{J}}(u):=\hat{\mathcal{J}}(\mathcal{S}(u), u)$ is the so-called reduced cost functional.

The solvability of (31) is ensured by the next theorem, whose proof adapts techniques given in [30] [31] and [17].

Theorem 3.2. There exists at least one optimal pair $(\bar{f}, \bar{u})$ that solves (29), such that $\bar{u}$ solves $(31)$ and $\bar{f}=\mathcal{S}(\bar{u})$.

Proof. The functional $\hat{\mathcal{J}}$ in (31) is bounded from below and therefore we can define $i:=\inf _{u \in \mathcal{U}_{a d}} \hat{\mathcal{J}}(u)$. Let $\left\{u_{n}\right\}_{n \in \mathbb{N}} \subset U_{a d}$ be a minimizing sequence, such that $\lim _{n \rightarrow \infty} J\left(u_{n}\right)=i$.

We have that $\mathcal{U}_{a d}$ is a convex, closed, and bounded subset of the reflexive Banach space $\mathcal{U}$. Hence, $\mathcal{U}_{a d}$ is weakly sequentially compact and we can extract a subsequence $\left\{u_{k}\right\}_{k \in \mathbb{N}} \subset\left\{u_{n}\right\}_{n \in \mathbb{N}}$ such that $u_{k} \rightarrow \bar{u} \in \mathcal{U}_{a d}$.

The weakly lower convergent sequence $\left\{u_{k}\right\}_{k \in \mathbb{N}}$ gives rise to the sequence $\left\{f_{k}\right\}_{k \in \mathbb{N}} \subset F \subset W$, defined by $f_{k}:=\mathcal{S}\left(u_{k}\right)$. Since the embedding $W \hookrightarrow L^{2}(I ; H)$ is compact, there exists a subsequence $\left\{f_{j}\right\}_{j \in \mathbb{N}} \subset\left\{f_{k}\right\}_{k \in \mathbb{N}}$ and $\bar{f} \in L^{2}(I ; H)$ such that $\left\{f_{j}\right\}_{j \in \mathbb{N}}$ converges strongly to $\bar{f}$. The fact $\bar{f} \in \mathcal{F}$ follows from standard arguments. Note that each couple $\left(f_{j}, u_{j}\right)$ satisfies $\mathcal{K}\left(f_{j}, u_{j}\right)=0$ by definition. Next, we want to pass to the limit in $\mathcal{K}\left(f_{j}, u_{j}\right)=0$.

Thanks to the estimate (18) in Proposition 2.3, the sequence $\left\{\partial_{t} f_{j}\right\}_{j \in \mathbb{N}}$ is bounded in $L^{2}\left(I, V^{*}\right)$ and therefore weakly convergent to $\partial_{t} \bar{f}$. Define $\bar{B}:=b(\bar{u})$. The boundedness of $B$ as in (7) and the smoothness of $b$ with respect to $u$ together with (18), ensures that $\left\{f(\nabla \cdot B)_{j}\right\}_{j \in \mathbb{N}}$ and $\left\{(B \cdot \nabla f)_{j}\right\}_{j \in \mathbb{N}}$ converge weakly to $\bar{f}(\nabla \cdot \bar{B})$ and $(\bar{B} \cdot \nabla \bar{f})$, respectively, where the norm in $L^{2}(I, H)$ has been considered. The weak convergence of $\left\{\mathcal{I} f_{j}\right\}_{j \in \mathbb{N}}$ to $\overline{\mathcal{I f}}$ follows from similar arguments [31]. These observations lead to the conclusion that the limit $\bar{f}$ solves (11), with $\bar{f}=\mathcal{S}(\bar{u})$. Therefore, the constraint $\mathcal{K}(\bar{f}, \bar{u})=0$ is satisfied.

Moreover, the convexity of $\mathcal{J}$ ensures that

$$
i=\liminf _{j} \mathcal{J}\left(f_{j}, u_{j}\right) \geq \mathcal{J}(\bar{f}, \bar{u})
$$

and therefore the pair $(\bar{f}, \bar{u})$ is a minimizer for the problem (29).

Remark 3.4. The uniqueness of the control $\bar{u}$ can not be stated a priori since $\hat{\mathcal{J}}$ is non convex.

\section{Two First-Order Optimality Systems}

We follow the standard approach [17] [23] [32] of characterizing the solution of our optimal control problem as the solution to first-order optimality conditions that constitute the optimality system.

Consider the reduced problem (31) and write the reduced functional $\hat{\mathcal{J}}$ as $\hat{\mathcal{J}}:=\mathcal{J}_{1}+\mathcal{J}_{2}, \quad \mathcal{J}_{i}: \mathcal{U} \rightarrow \mathbb{R}^{+}, \quad i=1,2$, where 


$$
\begin{aligned}
& \mathcal{J}_{1}(u):=D(\mathcal{S}(u))+\frac{v}{2}\|u\|_{2}^{2}, \\
& \mathcal{J}_{2}(u):=\gamma\|u\|_{1} .
\end{aligned}
$$

Remark 4.1. The functional $\mathcal{J}_{1}$ is smooth and possibly nonconvex, while $\mathcal{J}_{2}$ is convex and nonsmooth.

The following definitions are needed in order to determine the first-order optimality system. If $\hat{\mathcal{J}}$ is finite at a point $u$, the Fréchet subdifferential of $\hat{\mathcal{J}}$ at $u$ is defined as follows [32]. We have

$$
\partial \hat{\mathcal{J}}(u):=\left\{\varphi \in \mathcal{U}^{*}: \liminf _{v \rightarrow u} \frac{\hat{\mathcal{J}}(v)-\hat{\mathcal{J}}(u)-\langle\varphi, v-u\rangle}{\|v-u\|_{2}} \geq 0\right\},
$$

where $\mathcal{U}^{*}$ is the dual space of $\mathcal{U}$. Any element $\varphi \in \partial \hat{\mathcal{J}}(u)$ is called a subgradient. In our framework, we have

$$
\partial \hat{\mathcal{J}}(u)=\nabla \mathcal{J}_{1}(u)+\partial \mathcal{J}_{2}(u)
$$

since $\mathcal{J}_{1}$ is Fréchet differentiable at $u$; this follows from standard arguments [17] [30]. Moreover, for each $\alpha>0$, it holds that $\partial(\alpha \hat{\mathcal{J}})=\alpha \partial \hat{\mathcal{J}}$; see [26].

The following proposition gives a necessary condition for a local minimum of $\hat{\mathcal{J}}$.

Proposition 4.1. If $\hat{\mathcal{J}}=\mathcal{J}_{1}+\mathcal{J}_{2}$, with $\mathcal{J}_{1}$ and $\mathcal{J}_{2}$ given by (4.1), attains a local minimum in $\bar{u} \in \mathcal{U}_{a d}$, then

$$
0 \in \partial \hat{\mathcal{J}}(\bar{u})
$$

or equivalently

$$
-\nabla \mathcal{J}_{1}(\bar{u}) \in \partial \mathcal{J}_{2}(\bar{u})
$$

Proof. Since $\mathcal{U}_{a d}$ is convex, $\bar{u}+\theta(v-\bar{u}) \in \mathcal{U}_{a d}$, for each $v \in \mathcal{U}_{a d}$ and $\theta \in(0,1]$. Since $\bar{u}$ is a local minimum, we have

$$
\hat{\mathcal{J}}(\bar{u}) \leq \hat{\mathcal{J}}(\bar{u}+\theta(v-\bar{u})),
$$

for $v$ sufficiently close to $\bar{u}$. Exploiting the convexity of $\mathcal{J}_{2}$, we have

$$
\mathcal{J}_{1}(\bar{u}+\theta(v-\bar{u}))-\mathcal{J}_{1}(\bar{u})+\theta\left(\mathcal{J}_{2}(v)-\mathcal{J}_{2}(\bar{u})\right) \geq 0 .
$$

Dividing by $\theta$ and considering the limit $\theta \rightarrow 0$, we obtain

$$
\mathcal{J}_{2}(v)-J_{2}(\bar{u})+\left\langle\nabla \mathcal{J}_{1}(\bar{u}), v-\bar{u}\right\rangle \geq 0 .
$$

Dividing by $\|v-\bar{u}\|_{2}$ and considering the following limit

$$
\liminf _{v \rightarrow \bar{u}} \frac{\mathcal{J}_{2}(v)-\mathcal{J}_{2}(\bar{u})+\left\langle\nabla \mathcal{J}_{1}(\bar{u}), v-\bar{u}\right\rangle}{\|v-\bar{u}\|_{2}} \geq 0,
$$

we conclude that $-\nabla \mathcal{J}_{1}(\bar{u}) \in \partial \mathcal{J}_{2}(\bar{u})$, according to the definiton in (33).

By using results in [22] [32], we have that (34) implies that each $\lambda \in \partial \mathcal{J}_{2}(\bar{u})$, with $\bar{u}$ a local minimum, satisfies the following inequality 


$$
\left\langle\nabla \mathcal{J}_{1}(\bar{u})+\lambda, v-\bar{u}\right\rangle \geq 0 \text { for each } v \in \mathcal{U}_{a d} .
$$

Moreover, recalling the definition of $\mathcal{J}_{2}$ in (32) and exploiting the isomorphism $U^{*} \simeq U$, the inclusion $\lambda \in \partial \mathcal{J}_{2}(\bar{u})$ gives the following

$$
\lambda \in \Lambda_{\gamma}:=\left\{l \in \mathcal{L}^{2}(I):|l| \leq \gamma \text { a.e. on } I\right\} .
$$

A pointwise analysis of (35), which takes into account the definition (25) of the admissible controls, ensures the existence of two nonnegative functions $\bar{\lambda}_{a}, \bar{\lambda}_{b} \in \mathcal{U}^{*}$ that play the role of Lagrange multipliers [17]. The previous considerations lead to the following proposition, that states the optimality system for the reduced problem (31).

Proposition 4.2. The optimal solution $\bar{u}$ of the minimization problem (31) with $\hat{\mathcal{J}}=\mathcal{J}_{1}+\mathcal{J}_{2}$ defined in (32), is characterized by the existence of $\left(\bar{\lambda}, \bar{\lambda}_{a}, \bar{\lambda}_{b}\right) \in \Lambda_{\gamma} \times \mathcal{U}^{*} \times \mathcal{U}^{*}$ such that

$$
\left\{\begin{array}{l}
\nabla \mathcal{J}_{1}(\bar{u})+\bar{\lambda}-\bar{\lambda}_{a}+\bar{\lambda}_{b}=0 \\
\bar{\lambda}_{b} \geq 0, \quad u_{b}-\bar{u} \geq 0, \quad\left\langle\bar{\lambda}_{b}, u_{b}-\bar{u}\right\rangle=0 \\
\bar{\lambda}_{a} \geq 0, \quad \bar{u}-u_{a} \geq 0, \quad\left\langle\bar{\lambda}_{a}, \bar{u}-u_{a}\right\rangle=0 \\
\bar{\lambda}=\gamma \text { a.e. on }\{t \in I: \bar{u}(t)>0\} \\
|\bar{\lambda}| \leq \gamma \text { a.e. on }\{t \in I: \bar{u}(t)=0\} \\
\bar{\lambda}=-\gamma \text { a.e. on }\{t \in I: \bar{u}(t)<0\}
\end{array}\right.
$$

We refer to the last three conditions in (37) for the pair $(\bar{u}, \bar{\lambda})$ as the complementarity conditions.

The differentiability of $\mathcal{K}, \mathcal{J}_{1}$ and $\mathcal{S}$ with respect to $f$ and $u$ allows us to compute $\nabla \mathcal{J}_{1}(u)$ in (37) within the adjoint approach. By definition, for each $u \in \mathcal{U}$, we have

$$
\nabla \mathcal{J}_{1}(u)=v u+\left(\mathcal{S}^{\prime}(u)\right)^{*} D^{\prime}(\mathcal{S}(u)) .
$$

By considering the total derivative of $\mathcal{K}(\mathcal{S}(u), u)=0$, we have

$$
\mathcal{K}_{f}(\mathcal{S}(u), u) \mathcal{S}^{\prime}(u)+\mathcal{K}_{u}(\mathcal{S}(u), u)=0 .
$$

Therefore, we obtain

$$
\nabla \mathcal{J}_{1}(u)=v u-\mathcal{K}_{u}(\mathcal{S}(u), u)^{*}\left(\mathcal{K}_{f}(\mathcal{S}(u), u)^{*}\right)^{-1} D^{\prime}(\mathcal{S}(u))
$$

Defining the adjoint variable $p$ as the solution to the following adjoint problem

$$
\mathcal{K}_{f}(\mathcal{S}(u), u)^{*} p=-D^{\prime}(\mathcal{S}(u)),
$$

we obtain the following reduced gradient

$$
\nabla \mathcal{J}_{1}(u)=v u+\mathcal{K}_{u}(f, u)^{*} p .
$$

After some calculation, we have that (38) can be rewritten as the following adjoint system 


$$
\left\{\begin{array}{l}
-\partial_{t} p(x, t)=b(x ; u) \nabla p(x, t)+\sum_{i, j=1}^{n} a_{i j}(x) \partial_{i j}^{2} p(x, t)+\tilde{\mathcal{I}} p(x, t)+\alpha(x, t) \text { on } Q \\
p\left(x, t_{f}\right)=0 \text { on } \Omega \\
\nabla p(x, t) \cdot n(x, t)=0 \text { on } \partial \Omega \times I \\
p\left(x, t_{k}^{-}\right)=p\left(x, t_{k}^{+}\right)+\beta(x, k) \text { on } \Omega, \text { for each } k=1, \cdots, K,
\end{array}\right.
$$

where $\alpha$ and $\beta$ depend on the choice of $D$ in (27) and (28). When $D$ is given by (27), $\alpha(x, t)=0$ and $\beta(x, k)=-2 x\left(\xi_{k}-\int_{\Omega} s f\left(s, t_{k}\right) \mathrm{d} s\right)$, for each $k=1, \cdots, K$. On the other hand, when $D$ is given by (28), $\alpha(x, t)=2 x\left(\xi(t)-\int_{\Omega} s f(s, t) \mathrm{d} s\right)$ and $\beta(x, k)=0$.

The operator $\tilde{\mathcal{I}}$ is defined as follows

$$
\tilde{\mathcal{I}} p(x, t):=\lambda\left[\int_{\Omega} p(y, t) g(y, x) \mathrm{d} y-p(x, t)\right] \text { for each }(x, t) \in Q .
$$

The terminal boundary-value problem (40) admits a unique solution $p \in H^{2,1}(Q)$ thanks to the assumptions (13) and (14), following the same arguments as in Theorem $2.1[29]$.

The reduced gradient in (39), for given $u, f$, and $p$, takes the following form

$$
\nabla \hat{\mathcal{J}}_{1}(u)=v u+\int_{\Omega} f(\nabla p) \cdot\left(\partial_{u} b\right) .
$$

The complementarity conditions in (37) can be recast in a more compact form, as follows. We define $\bar{\mu}:=\bar{\lambda}-\bar{\lambda}_{a}+\bar{\lambda}_{b}$. For each $k \in \mathbb{R}^{+}$, we define the following quantity

$$
\begin{aligned}
E(\bar{u}, \bar{\mu}):= & \bar{u}-\max \{0, \bar{u}+k(\bar{\mu}-\gamma)\}-\min \{0, \bar{u}+k(\bar{\mu}+\gamma)\} \\
& +\max \left\{0, \bar{u}-u_{b}+k(\bar{\mu}-\gamma)\right\}+\min \left\{0, \bar{u}-u_{a}+k(\bar{\mu}+\gamma)\right\} .
\end{aligned}
$$

The complementarity conditions in (37) and the inequalities related to the Lagrange multipliers $\bar{\lambda}_{a}$ and $\bar{\lambda}_{b}$, together with the requirement $\bar{\lambda} \in \Lambda_{\gamma}$, are equivalent to $E(\bar{u}, \bar{\mu})=0$; see, e.g., [22].

The previous considerations can be summarized in the following propositions.

Proposition 4.3. (Optimality system for a discrete-in-time tracking functional)

A local solution $(f, u) \in \mathcal{F} \times \mathcal{U}_{a d}$ of (29) with $D$ given by (27) is characterized by the existence of $(p, \mu) \in H^{2,1}(Q) \times \mathcal{U}^{*}$, such that the following system is satisfied

$$
\begin{cases}v u+\int_{\Omega} f(\nabla p) \cdot\left(\partial_{u} b\right)+\mu=0 & \text { a.e. in } I \\ \partial_{t} f(x, t)=\nabla \cdot F(x, t)+\mathcal{I}(f(x, t)) & \text { for }(x, t) \in Q \\ f(x, 0)=f_{0}(x) & \text { for } x \in \Omega \\ F(x, t) \cdot n(x, t)=0 & \text { for }(x, t) \in \Sigma \\ -\partial_{t} p(x, t)=b(x ; u) \nabla p(x, t)+\sum_{i, j=1}^{n} a_{i j}(x) \partial_{i j}^{2} p(x, t)+\tilde{\mathcal{I}} p(x, t) & \text { for }(x, t) \in Q \\ p\left(x, t_{f}\right)=0 & \text { for } x \in \Omega \\ \nabla p(x, t) \cdot n(x, t)=0 & \text { for }(x, t) \in \Sigma \\ p\left(x, t_{k}^{-}\right)=p\left(x, t_{k}^{+}\right)-2 x\left(\xi_{k}-\int_{\Omega} s f\left(s, t_{k}\right) \mathrm{d} s\right) & \text { for } x \in \Omega, k=1, \cdots, K \\ E(u, \mu)=0 & \text { a.e. in } I .\end{cases}
$$


Proposition 4.4. (Optimality system for a continuous-in-time tracking functional)

A local solution $(f, u) \in \mathcal{F} \times \mathcal{U}_{a d}$ of (29) with $D$ given by (28) is characterized by the existence of $(p, \mu) \in H^{2,1}(Q) \times \mathcal{U}^{*}$, such that the following system is satisfied

$$
\begin{cases}v u+\int_{\Omega} f(\nabla p) \cdot\left(\partial_{u} b\right)+\mu=0 & \text { a.e. in } I \\ \partial_{t} f(x, t)=\nabla \cdot F(x, t)+\mathcal{I}(f(x, t)) & \text { for }(x, t) \in Q \\ f(x, 0)=f_{0}(x) & \text { for } x \in \Omega \\ F(x, t) \cdot n(x, t)=0 & \text { for }(x, t) \in \Sigma \\ -\partial_{t} p(x, t)=b(x ; u) \nabla p(x, t)+\sum_{i, j=1}^{n} a_{i j}(x) \partial_{i j}^{2} p(x, t)+\tilde{\mathcal{I}} p(x, t) & \\ \quad+2 x\left(\xi(t)-\int_{\Omega} s f(s, t) \mathrm{d} s\right) & \text { for }(x, t) \in Q \\ p\left(x, t_{f}\right)=0 & \text { for } x \in \Omega \\ \nabla p(x, t) \cdot n(x, t)=0 & \text { for }(x, t) \in \Sigma \\ E(u, \mu)=0 & \text { a.e. in } I .\end{cases}
$$

\section{Numerical Approximation of the Optimality Systems}

In this section, we discuss the discretization of the optimality systems given in (42) and (43). For simplicity, we focus on a one-dimensional case with $\Omega:=(r, s) \subset \mathbb{R}$. We define $h:=\frac{s-r}{N-1}, N \in \mathbb{N}, N>1$, and $\delta t:=\frac{t_{f}-t_{0}}{M}, M \in \mathbb{N}$. The space and time grids are defined as follows

$$
\begin{aligned}
& \Omega_{h}:=\left\{x_{j}=r+(j-1) h, j=1, \cdots, N\right\}, \\
& I_{\delta t}:=\left\{t_{n}=t_{0}+n \delta t, n=0, \cdots, M\right\} .
\end{aligned}
$$

Notice that a cell-centered space discretization is considered with cells midpoints at $x_{j}, j=1, \cdots, N$, and cell faces at $x_{j \pm h / 2}$.

The approximation of the forward and backward FP PIDEs is based on a discretization method discussed in [33], where a convergent and conservative numerical scheme for solving the FP problem of a JD process is presented. This discretization scheme is obtained based on the so-called method of lines (MOL) [34]. The differential operator in (11) is discretized by applying the Chang-Cooper (CC) scheme [35] [36]. Setting $w_{j}(t):=h B_{j+\frac{1}{2}}(t) / a_{j+\frac{1}{2}}$, and $\delta_{j}(t):=1 / w_{j}(t)-1 /\left(\exp \left\{w_{j}(t)\right\}-1\right)$, the discretization of the differential operator is carried out as follows

$$
\partial_{x} F\left(x_{j}, t\right) \approx \frac{F_{j+\frac{1}{2}}(t)-F_{j-\frac{1}{2}}(t)}{h}, \quad j=1, \cdots, N,
$$

where

$$
F_{j+\frac{1}{2}}(t)=B_{j+\frac{1}{2}}(t)\left[\delta_{j}(t) f_{j}(t)+\left(1-\delta_{j}(t)\right) f_{j+1}(t)\right]+a_{j+\frac{1}{2}} \frac{f_{j+1}(t)-f_{j}(t)}{h} .
$$

The zero-flux boundary conditions are implemented referring to the points $x_{\frac{1}{2}}$ and 
$X_{N+\frac{1}{2}}$. The integral addend is approximated by the midpoint rule. After spacial discretization, the forward FP PIDE problem takes the following form

$$
f^{\prime}(t)=(\mathcal{A}+\mathcal{G}) f(t),
$$

where $f(t) \in \mathbb{R}^{N}$. The matrices $\mathcal{A}$ and $\mathcal{G}$ correspond to the CC scheme and to the quadrature rule, respectively. The time integration of (44) is carried out with the combination of the Strang-Marchuk (SM) splitting scheme [37] [38] together with a predictor-corrector scheme [39]. We refer to [40] for a detailed introduction to splitting methods. With this choice, the numerical scheme solving (11) is second-order convergent both in space and time with respect to the $L_{h, \delta t}^{2}$ norm. Notice that the chosen numerical method for the FP problem must ensure that the PDF solution is nonnegative and that the total probability remains constant along the time evolution. See [33] for all details and numerical analysis results.

If we follow the optimize-before-discretize (OBD) approach, the optimality system has already been computed on a continuous level as in (42) and (43) and subsequently discretized. As a consequence, the OBD approach allows one to discretize the forward abd adjoint FP problems according to different numerical schemes. However, the OBD procedure might introduce an inconsistency between the discretized objective and the reduced gradient; see [15] and references therein. For this reason, the DBO (discretize-before-optimize) approach could be preferred and we pursue it in this work.

The DBO approach results in the following approximations

$$
\begin{aligned}
& \partial_{x} p\left(x_{j}, t\right) \approx\left(1-\delta_{j-1}(t)\right) \frac{p_{i-1}(t)-p_{i}(t)}{h}+\delta_{j}(t) \frac{p_{i}(t)-p_{i+1}(t)}{h} \\
& \partial_{x x}^{2} p\left(x_{j}, t\right) \approx \frac{p_{i+1}(t)-2 p_{i}(t)+p_{i-1}(t)}{h^{2}}
\end{aligned}
$$

together with the midpoint quadrature formula applied to $\tilde{\mathcal{I}}$ in (40). We have the following semi-discretized system

$$
-p^{\prime}(t)=\left(\mathcal{A}^{\mathrm{T}}+\mathcal{G}^{\mathrm{T}}\right) p(t), \quad p(t) \in \mathbb{R}^{N} .
$$

The time integration of (45) is carried out with the combination of the SM splitting with a predictor corrector scheme, as in (44).

\section{A Proximal Optimization Scheme}

In this section, we discuss a proximal optimization scheme for solving (31). This scheme and the related theoretical discussion follow the work in [19] [27]. Proximal methods conveniently exploit the additive structure of the reduced objective, and in our framework, we have that the reduced functional $\hat{\mathcal{J}}$ is given by the sum of a nonconvex smooth function $\mathcal{J}_{1}$ and a convex nonsmooth function $\mathcal{J}_{2}$ as in (32).

For our discussion, we need the following definitions and properties.

Definition 6.1. Let $Z$ be a Hilbert space and 1 a convex lower semi continuous function, $l: Z \rightarrow \mathbb{R}$. The proximity operator prox $_{l}: Z \rightarrow Z$ of 1 is defined as follows 


$$
\operatorname{prox}_{l}(z):=\underset{w \in Z}{\arg \min }\left\{l(w)+\frac{1}{2}\|z-w\|_{Z}^{2}\right\}, \quad z \in Z .
$$

Proposition 6.1. Let $Z$ be a Hilbert space and 1 a convex lower semi continuous function, $l: Z \rightarrow \mathbb{R}$, with proximity operator prox $_{l}$. The following relation holds

$$
p=\operatorname{prox}_{l}(z) \Leftrightarrow z-p \in \partial l(p),
$$

where $\partial l$ is the subdifferential defined in (33).

Proof. See [27].

Proposition 6.2. The solution $\bar{u}$ of (31) satisfies

$$
\bar{u}=\operatorname{prox}_{\alpha \mathcal{J}_{2}}\left(\bar{u}-\alpha \nabla \mathcal{J}_{1}(\bar{u})\right)
$$

for each $\alpha>0$.

Proof. From Proposition 4.7 and by using (46), we have

$$
\begin{aligned}
\bar{u} \text { solves (3.8) } & \Rightarrow-\nabla \mathcal{J}_{1}(\bar{u}) \in \partial \mathcal{J}_{2}(\bar{u}) \\
& \Leftrightarrow\left(\bar{u}-\alpha \nabla \mathcal{J}_{1}(\bar{u})\right)-\bar{u} \in \alpha \partial \mathcal{J}_{2}(\bar{u}) \\
& \Leftrightarrow \bar{u}=\operatorname{prox}_{\alpha \mathcal{J}_{2}}\left(\bar{u}-\alpha \nabla \mathcal{J}_{1}(\bar{u})\right)
\end{aligned}
$$

The relation (47) suggests that a solution procedure based on a fixed point iteration should be pursued. We discuss how such algorithm can be implemented.

In the following, we assume that $\mathcal{J}_{1}(u)$ in (32) has a locally Lipschitz-continuous gradient $\nabla \mathcal{J}_{1}$ as follows

$$
\left\|\nabla \mathcal{J}_{1}(u)-\nabla \mathcal{J}_{1}(v)\right\| \leq L\|u-v\|,
$$

for each $v \in \mathcal{V}, \mathcal{V} \subset \mathcal{U}_{a d}$ neighborhood of $u$, with $L$ a Lipschitz continuity constant. It is shown in [28] that (48) implies the following inequality

$$
\mathcal{J}_{1}(u) \leq \mathcal{J}_{1}(v)+\left\langle\nabla \mathcal{J}_{1}(v), u-v\right\rangle+\frac{L}{2}\|u-v\|^{2},
$$

for each $v \in \mathcal{V}$, and hence

$$
\min _{u \in \mathcal{U}_{a d}}\left\{\mathcal{J}_{1}(u)+\mathcal{J}_{2}(u)\right\} \leq \min _{u \in \mathcal{U}_{a d}}\left\{\mathcal{J}_{1}(u)+\mathcal{J}_{2}(u)+\left\langle\nabla \mathcal{J}_{1}(v), u-v\right\rangle+\frac{L}{2}\|u-v\|^{2}\right\} .
$$

Inequality (49) is the starting point for the formulation of a proximal scheme, whose strategy consists of minimizing the right-hand side in (49). One can prove the following equality

$$
\begin{aligned}
& \min _{u \in \mathcal{U}_{a d}}\left\{\mathcal{J}_{1}(u)+\mathcal{J}_{2}(u)+\left\langle\nabla \mathcal{J}_{1}(v), u-v\right\rangle+\frac{L}{2}\|u-v\|^{2}\right\} \\
& =\min _{u \in \mathcal{U}_{a d}}\left\{\mathcal{J}_{2}(u)+\frac{L}{2}\left\|u-\left(v-\frac{1}{L} \nabla \mathcal{J}_{1}(v)\right)\right\|^{2}\right\} .
\end{aligned}
$$

Recall the definition of $\mathcal{J}_{2}$ in (32). The following lemma gives an explicit expression for the right-hand side in (50).

Lemma 6.3. Let $\mathcal{U}_{a d}$ be as in (25). Then 


$$
\underset{u \in \mathcal{U}_{a d}}{\arg \min }\left\{\tau\|u\|_{1}+\frac{1}{2}\|u-w\|^{2}\right\}=\mathbb{S}_{\tau}^{\mathcal{U}_{a d}}(w) \text { for each } w \in \mathcal{U},
$$

where the projected soft thresholding function $\mathbb{S}_{\tau}^{\mathcal{U}_{a d}}$ is defined as follows

$$
\mathbb{S}_{\tau}^{\mathcal{U}_{a d}}(w):= \begin{cases}\min \left\{w-\tau, u_{b}\right\} & \text { on }\{t \in I: w(t)>\tau\} \\ 0 & \text { on }\{t \in I:|w(t)| \leq \tau\} \\ \max \left\{w+\tau, u_{a}\right\} & \text { on }\{t \in I: w(t)<-\tau\} .\end{cases}
$$

Proof. See [19].

Based on this lemma, we conclude the following

$$
\underset{u \in \mathcal{U}_{a d}}{\arg \min }\left\{\mathcal{J}_{2}(u)+\frac{L}{2}\left\|u-\left(v-\frac{1}{L} \nabla \mathcal{J}_{1}(v)\right)\right\|^{2}\right\}=\underset{\frac{\gamma}{L}}{\mathcal{U}_{a d}}\left(v-\frac{1}{L} \nabla \mathcal{J}_{1}(v)\right),
$$

which can be taken as starting point for a fixed-point algorithm as follows

$$
u_{k+1}=\mathbb{S}_{\frac{\gamma}{L}}^{\mathcal{u}_{a d}}\left(u_{k}-\frac{1}{L_{k}} \nabla \mathcal{J}_{1}\left(v_{k}\right)\right)
$$

where $L_{k}$ is the local Lipschitz continuity constant defined in (48). Such method has been investigated in [19] [25] [27]. In this work, we apply an extension of (51), which takes for each iteration $\mathrm{k}$ the following form

$$
u_{k+1}=\mathbb{S}_{\gamma / L_{k}}^{\mathcal{u}_{a d}}\left(u_{k}-\frac{1}{L_{k}} \nabla \mathcal{J}_{1}\left(u_{k}\right)+\theta_{k}\left(u_{k}-u_{k-1}\right)\right) \text {, }
$$

with $\theta_{k} \in(0,1)$. This method has been proposed in [28]. Our inertial proximal method is summarized in the following algorithm.

\section{Algorithm 1 (Inertial proximal method).}

Input: initial guess $u_{0}, i=0, i_{\max }, \theta_{i} \in(0,1)$, tolerance tol, Lipschitz constant $L$.

1) While $i \leq i_{\max }$, do:

(a) Evaluate $\nabla \mathcal{J}_{1}\left(u_{i}\right)$ according to Algorithm 2.

(b) Update $L_{i}=\eta L_{i}$ until

$$
\mathcal{J}_{1}(\tilde{u}) \leq \mathcal{J}_{1}\left(u_{i}\right)+\left\langle\nabla_{u} \mathcal{J}_{1}\left(u_{i}\right), \tilde{u}-u_{i}\right\rangle+\frac{L}{2}\left\|\tilde{u}-u_{i}\right\|^{2}
$$

where

$$
\tilde{u}=\mathbb{S}_{\gamma / L_{i}}^{\mathcal{u}_{a d}}\left(u_{i}-\frac{1}{L_{i}} \nabla \mathcal{J}_{1}\left(u_{i}\right)+\theta_{i}\left(u_{i}-u_{i-1}\right)\right)
$$

(c) Set $u_{i+1}=\tilde{u}$.

(d) Compute $E$ according to (42) or (43).

(e) If $E<$ tol, break.

(f) $i=i+1$.

Remark 6.1. The backtracking scheme in Algorithm 1 provides an estimation of the upper bound of the Lipschitz constant in (48), since it is not known a priori. The initial guess for $L$ is chosen as follows. Given a small variation $\varepsilon$ of $u$, we have 


$$
L=\max \left\{\frac{\left\|\nabla \mathcal{J}_{1}(u)-\nabla \mathcal{J}_{1}(u+\varepsilon)\right\|_{2}}{\|\varepsilon\|_{2}}, \frac{\left\|\nabla \mathcal{J}_{1}(u)-\nabla \mathcal{J}_{1}(u-\varepsilon)\right\|_{2}}{\|\varepsilon\|_{2}}\right\} .
$$

\section{Algorithm 2 (Evaluation of the gradient).}

Input: $u_{i}$, initial value $f_{0}$ at time $t_{0}$, terminal value $p_{f}$ at time $t_{f}$.

1) Compute $f_{i}$, given $f_{0}$ and $u_{i}$.

2) Compute $p_{i}$.

3) Evaluate $\nabla \mathcal{J}_{1}\left(u_{i}\right)$ according to (41).

Next, we discuss the convergence of our algorithm, using some existing results [28] [41].

Proposition 6.4. The sequence $\left\{u_{k}\right\}_{k \in \mathbb{N}}$ generated by (52) satisfies the following properties.

- The sequence $\left\{J\left(u_{k}\right)\right\}_{k \in \mathbb{N}}$ converges in $\mathbb{R}$.

- There exists a weakly convergent subsequence $\left\{u_{k_{j}}\right\}_{j \in \mathbb{N}} \subset\left\{u_{k}\right\}_{k \in \mathbb{N}}$.

Definition 6.2. The proximal residual $r$ is defined as follows

$$
r(u):=u-\mathbb{S}_{\gamma}^{u_{a d}}\left(u-\nabla \mathcal{J}_{1}(u)\right)
$$

Proposition 6.12 tells us that $r(u)=0$ in $L^{2}(I)$ whenever $u$ solves (31). The next proposition establish a connection between the condition $r(u)=0$ and the solution provided by Algorithm 1; see, e.g., [19].

Proposition 6.5. Let $\left\{u_{k}\right\}_{k \in \mathbb{N}}$ be the sequence generated by Algorithm 1. Then the following holds

$$
\min _{0 \leq k \leq K}\left\|r\left(u_{k}\right)\right\|_{2}^{2}=\mathcal{O}(1 / K)
$$

\section{Numerical Experiments}

In this section, we present results of numerical experiments to validate the performance of our optimal control framework. Our purpose is to determine a sparse control $u=u(t)$ such that the expected value of the process X defined by (1) minimizes the quantity defined by (27) and (28).

We implement the discretization scheme and the algorithm described in Section 5. We take $\Omega=(-100,100)$ and $\left[t_{0}, t_{f}\right]=[0,1]$, and assume that the initial probability density function $f_{0}$ is given, $f_{0} \sim \mathcal{N}(0,10)$. The compound Poisson process corresponds to the choice $\lambda=3$ and $g \sim \mathcal{N}(0,0.04)$. We take $b(x, u):=x-u(t)$ and $\sigma(x)=50$. In case of (27), we consider $\xi=[-5,20,0,10,-10,0,-5,20]$. In the case of (28), we take $\xi(t)=20 \sin (10 t)$. We choose $N=300$ and $M=200$.

In the first series of experiments, we consider the setting with $v=10^{-8}$ and $\gamma=0$ in (32). Further, we do consider constraints on the control. Corresponding to this choice and to the discrete-in-time tracking functional (27), we report in Figure 1 and Figure 2 the solution for the state and the adjoint variables, respectively. On the other hand, using the continuous-in-time tracking functional (28), we obtain the state and the adjoint variables depicted in Figure 3 and Figure 4, respectively.

Also for the case $\gamma=0$ and both tracking functionals, we report in Table 1 and 


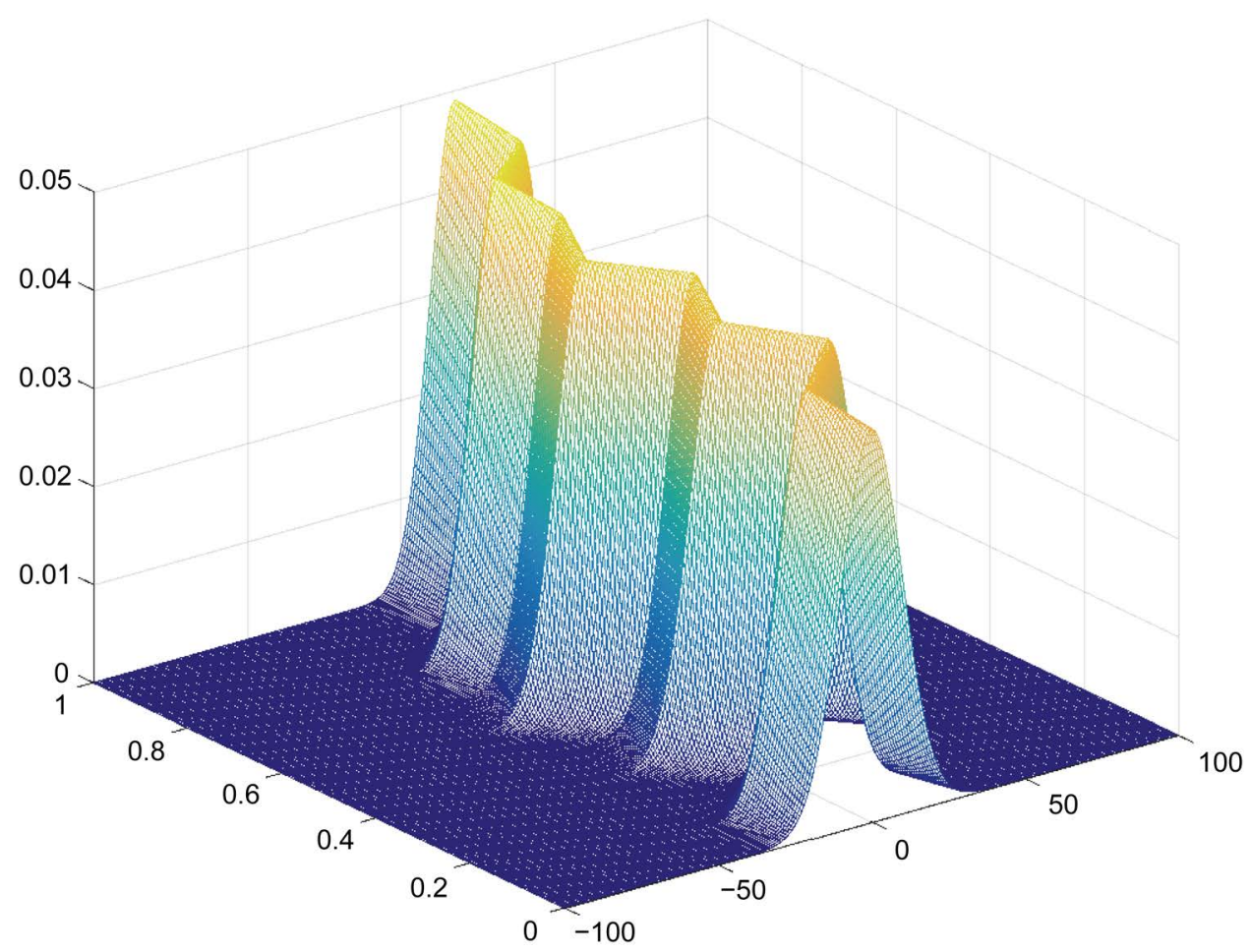

Figure 1. State variable in the case of the discrete-in-time tracking functional defined in (27), with $\gamma=0$.

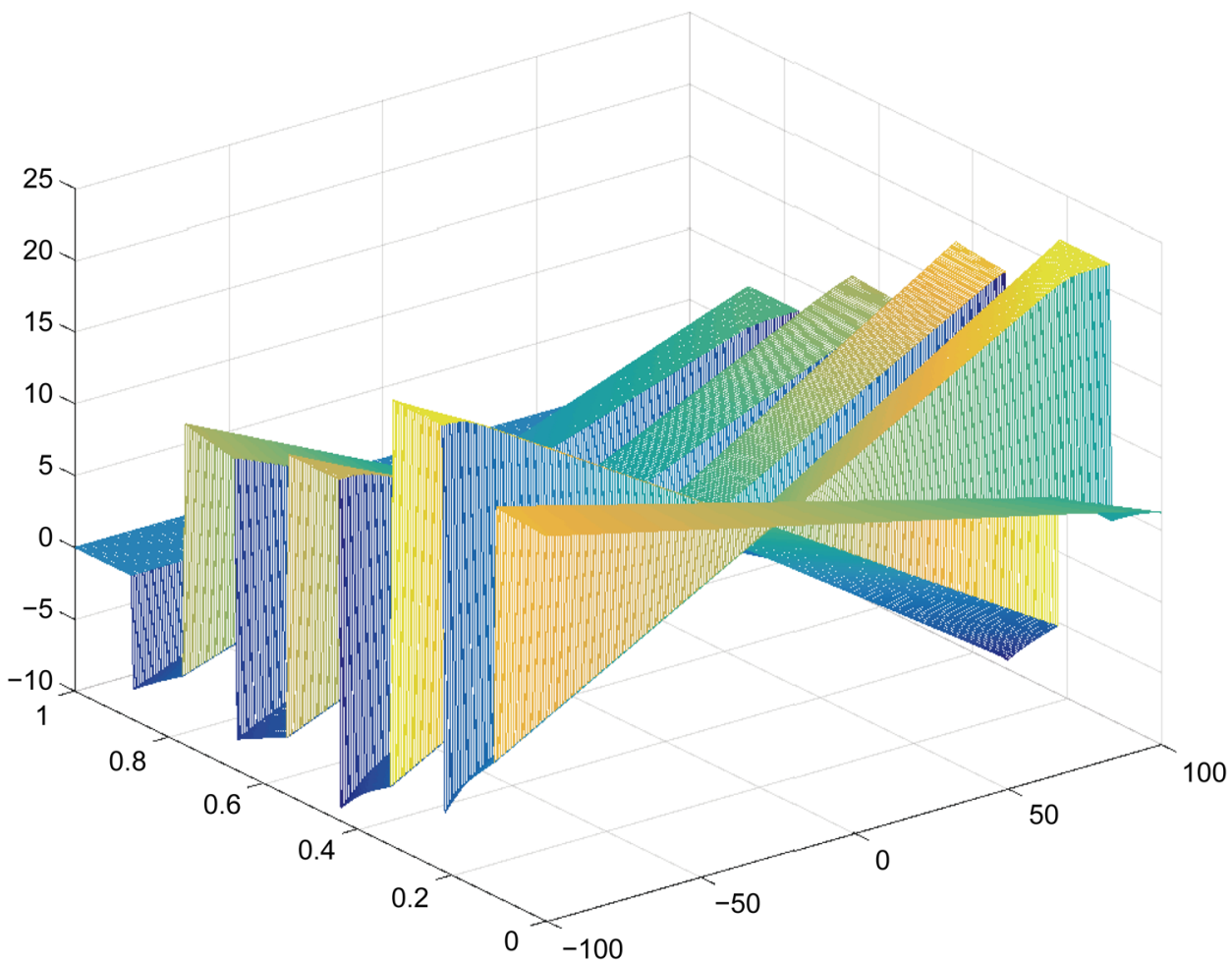

Figure 2. Adjoint variable in case of the discrete-in-time tracking functional defined in (27), with $\gamma=0$. 


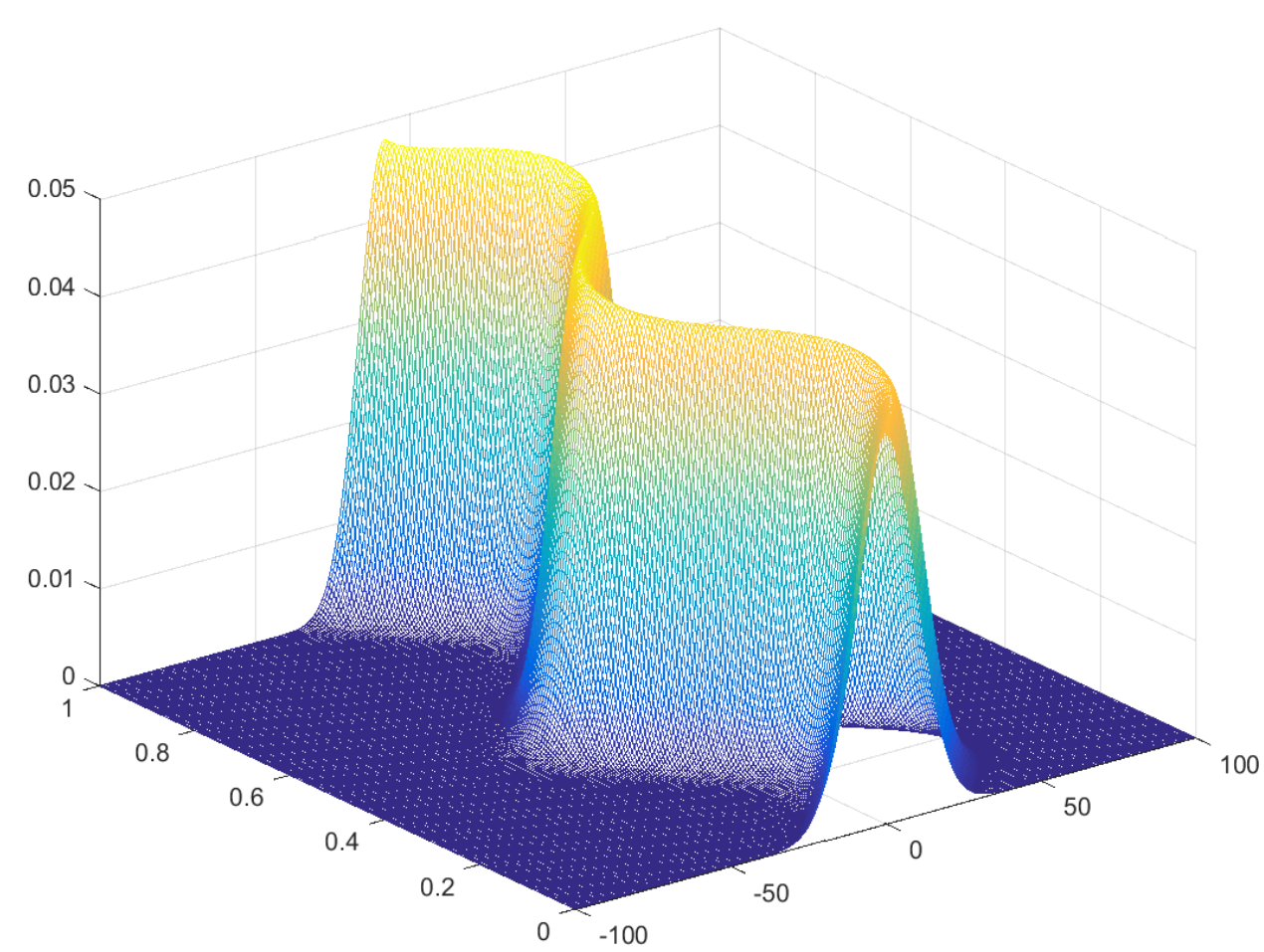

Figure 3. State variable in the case of the continuous-in-time tracking functional defined in (28), with $\gamma=0$.

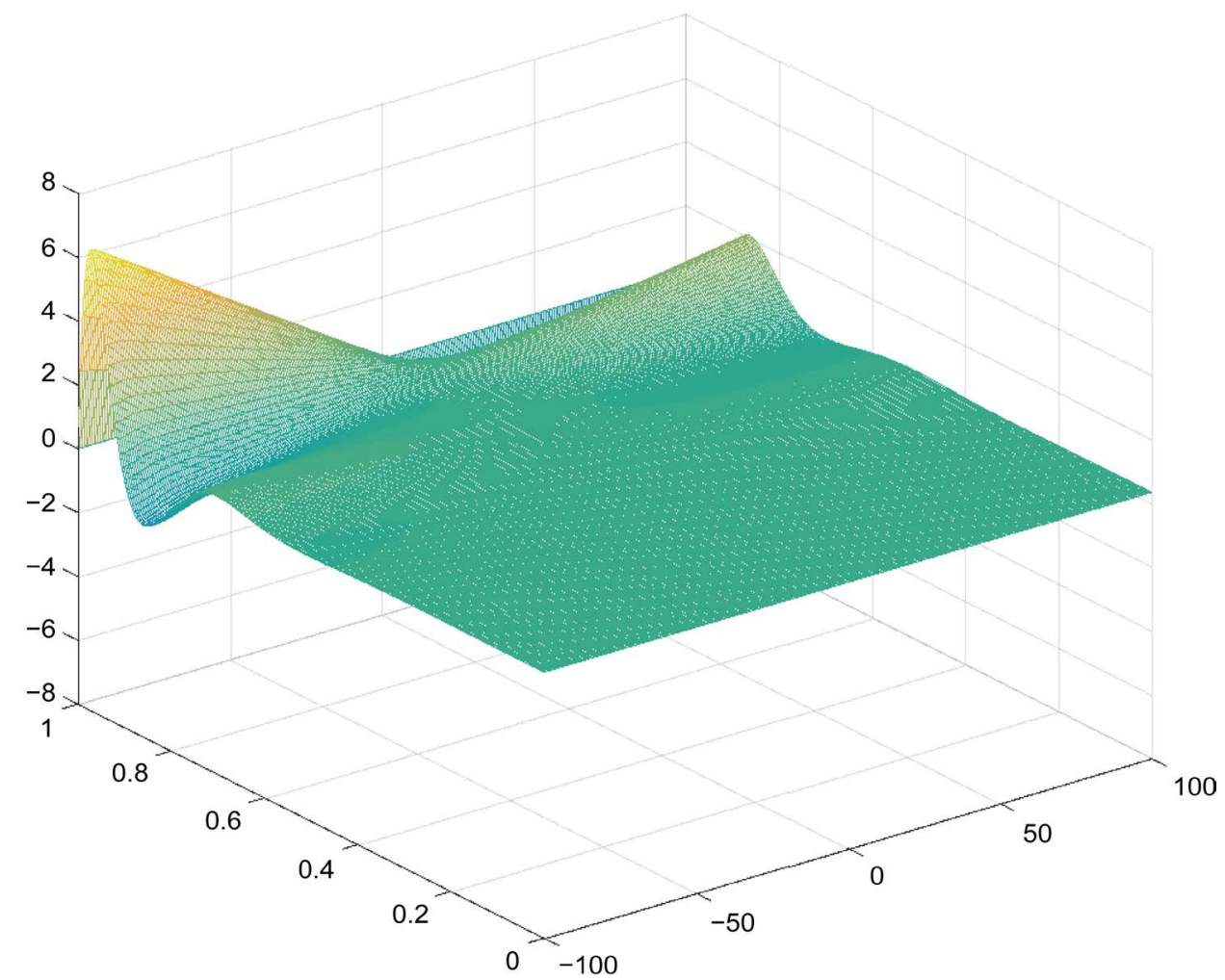

Figure 4. Adjoint variable in case of the continuous-in-time tracking functional defined in (28), with $\gamma=0$. 
Table 2 the values of the tracking error for different values of the weight $v$. As expected, the tracking improves as the value of this optimization parameter becomes smaller. In Figure 1 and Figure 3, we can see that the optimal control $u$ drives the expected mean value of the PDF towards the mean values given by $\xi_{k}$ and $\xi(t)$, respectively.

Next, we investigate the behavior of the optimal solution considering the full optimization setting, that is, the case when the $L^{1}$-cost actively enters in the optimization process, i.e. $\gamma>0$, and the control is constrained by the bounds $u_{a}=-10, u_{b}=10$ in (25). For simplicity, we discuss only the case with $v=10^{-2}$.

In Figures 5-7, we depict the optimal controls for three different choices of values of $\gamma$ and considering the discrete-in-time tracking functional given by (27). In Figures 8-10, we show the optimal controls for three different choices of values of $\gamma$ and considering the continuous-in-time tracking functional given by (28). In both cases, we can clearly see that increasing the value of the parameter $\gamma$ significantly increases the sparsity of the solution, as expected.

Finally, in the Table 1 and Table 2, we also report values of the tracking error when both the $L^{2}$ - and $L^{1}$-costs are considered. For a direct comparison with the first series of experiments, we consider an unconstrained control. We find that already with a small value of $\gamma$, the tracking ability of the optimization scheme worsen for both choices of the tracking functional.

Table 1. Tracking error of the discrete-in-time functional $D(f)$ given by (27).

\begin{tabular}{ccc}
\hline$D(f)$ & $v$ & $\gamma$ \\
\hline $2.02 \times 10^{-5}$ & $10^{-10}$ & 0 \\
$2.49 \times 10^{-5}$ & $10^{-6}$ & 0 \\
$1.34 \times 10^{-2}$ & $10^{-4}$ & 0 \\
$1.12 \times 10^{-2}$ & $10^{-10}$ & $10^{-4}$ \\
$1.27 \times 10^{-2}$ & $10^{-6}$ & $10^{-4}$ \\
$2.61 \times 10^{-2}$ & $10^{-4}$ & $10^{-4}$ \\
\hline
\end{tabular}

Table 2. Tracking error of the continuous-in-time functional $D(f)$ given by (28).

\begin{tabular}{ccc}
\hline$D(f)$ & $v$ & $\gamma$ \\
\hline $7.78 \times 10^{-4}$ & $10^{-10}$ & 0 \\
$1.05 \times 10^{-3}$ & $10^{-6}$ & 0 \\
$1.71 \times 10^{-2}$ & $10^{-4}$ & 0 \\
$7.96 \times 10^{-2}$ & $10^{-10}$ & $10^{-4}$ \\
$1.38 \times 10^{-1}$ & $10^{-6}$ & $10^{-4}$ \\
$1.23 \times 10^{-1}$ & $10^{-4}$ & $10^{-4}$ \\
\hline
\end{tabular}




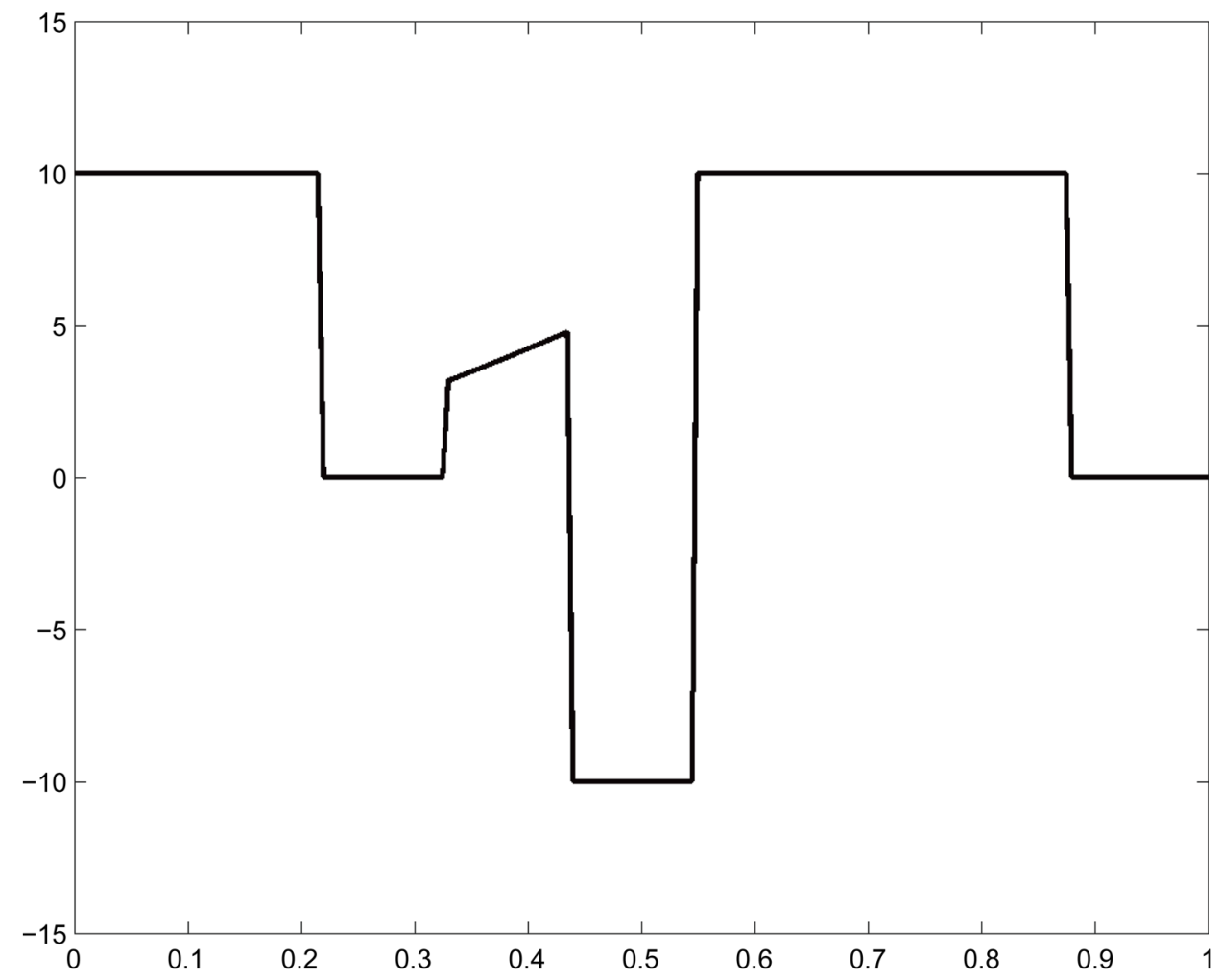

Figure 5. Optimal control with $\gamma=1 / 2$ and tracking objective given by (27).

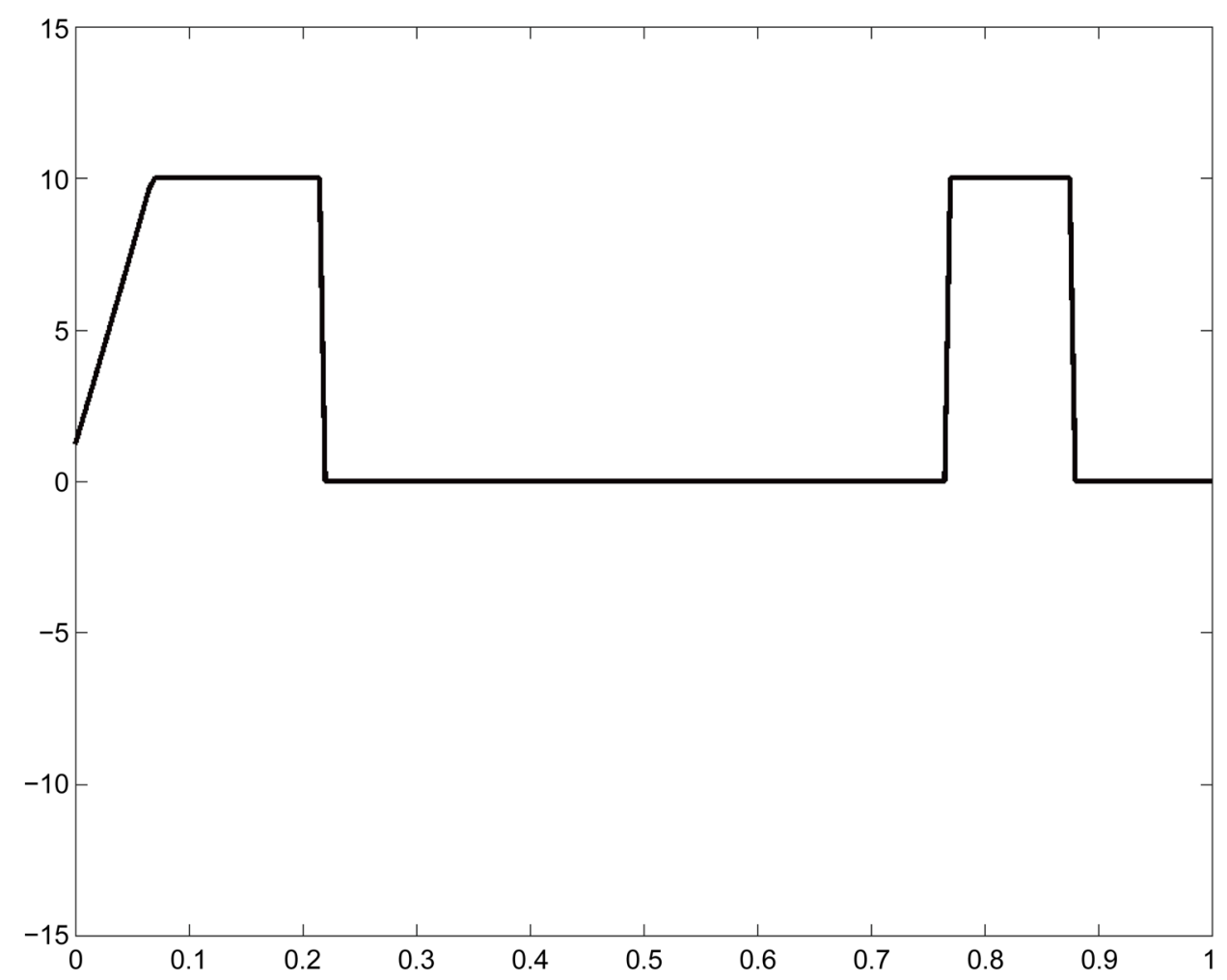

Figure 6. Optimal control with $\gamma=3$ and tracking objective given by (27). 


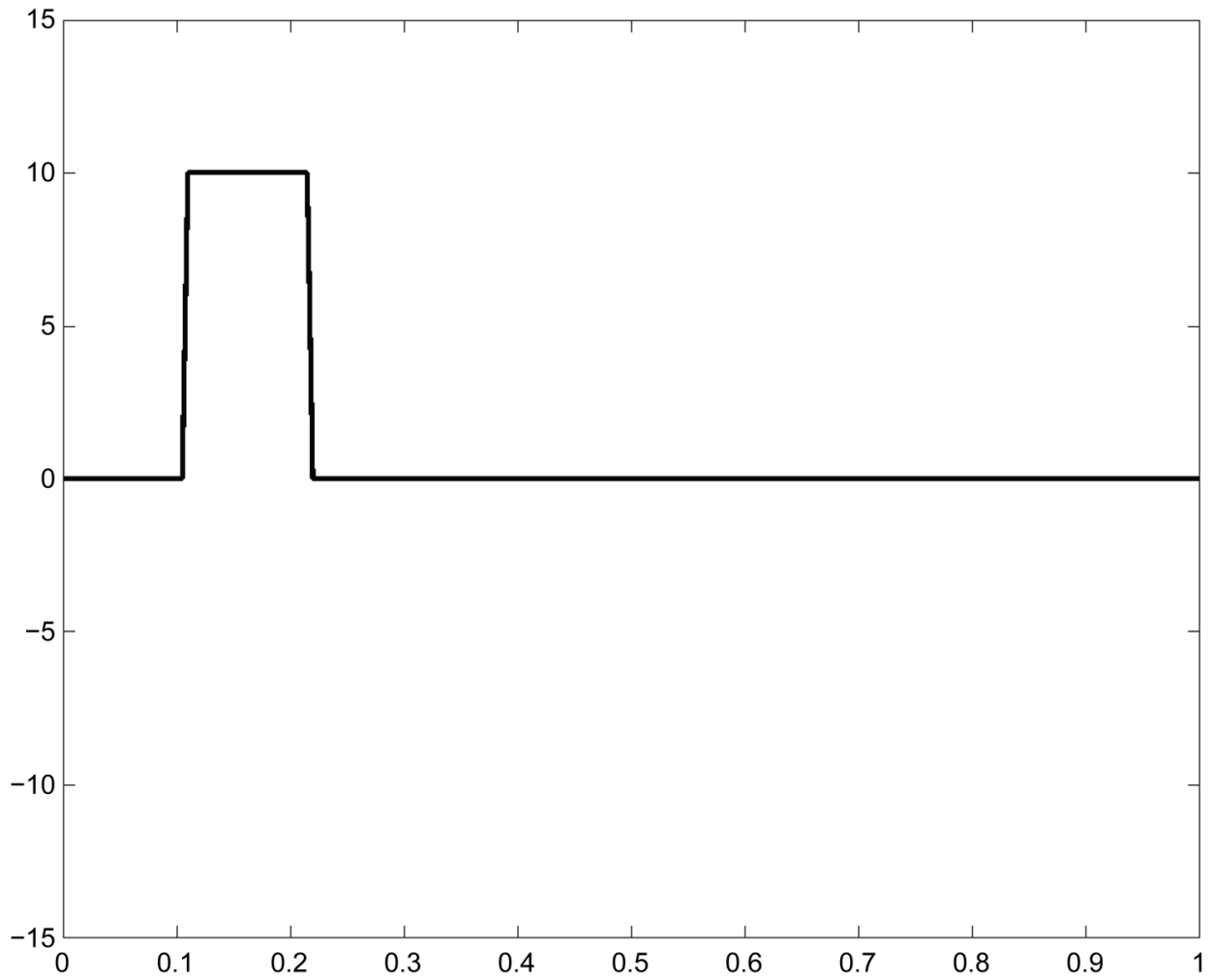

Figure 7. Optimal control with $\gamma=5$ and tracking objective given by (27).

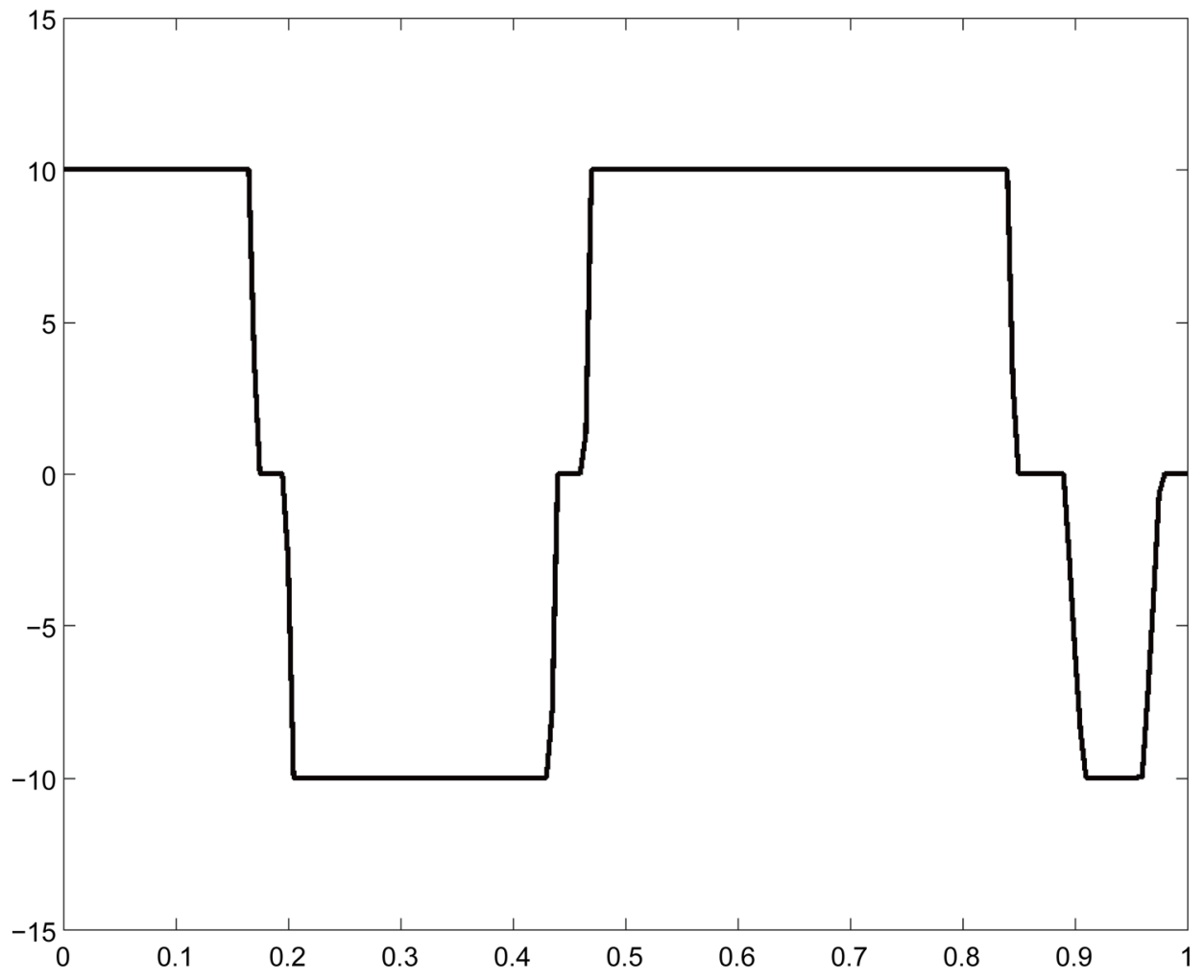

Figure 8. Optimal control with $\gamma=1 / 2$ and tracking objective given by (28). 


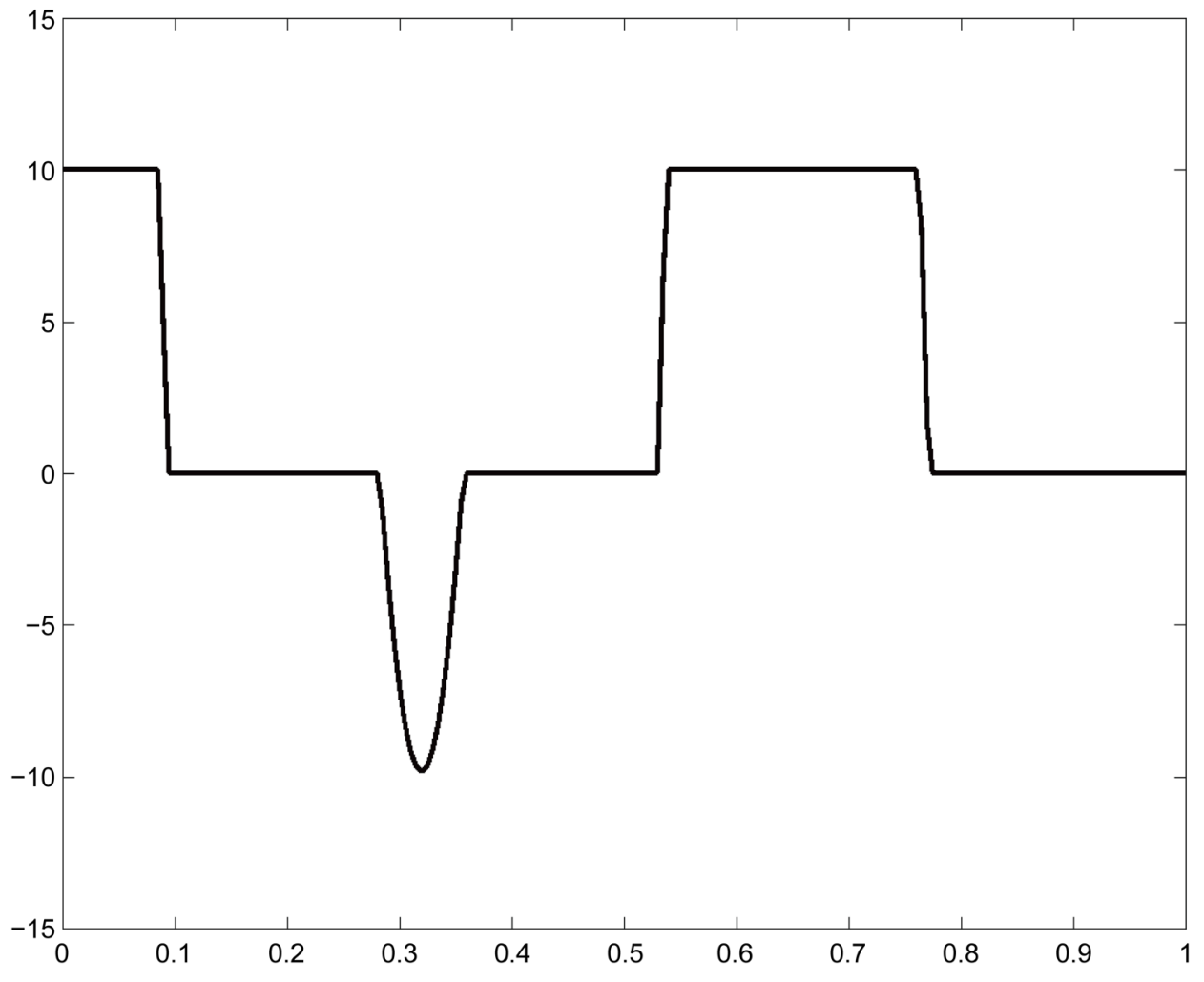

Figure 9. Optimal control with $\gamma=3$ and tracking objective given by (28).

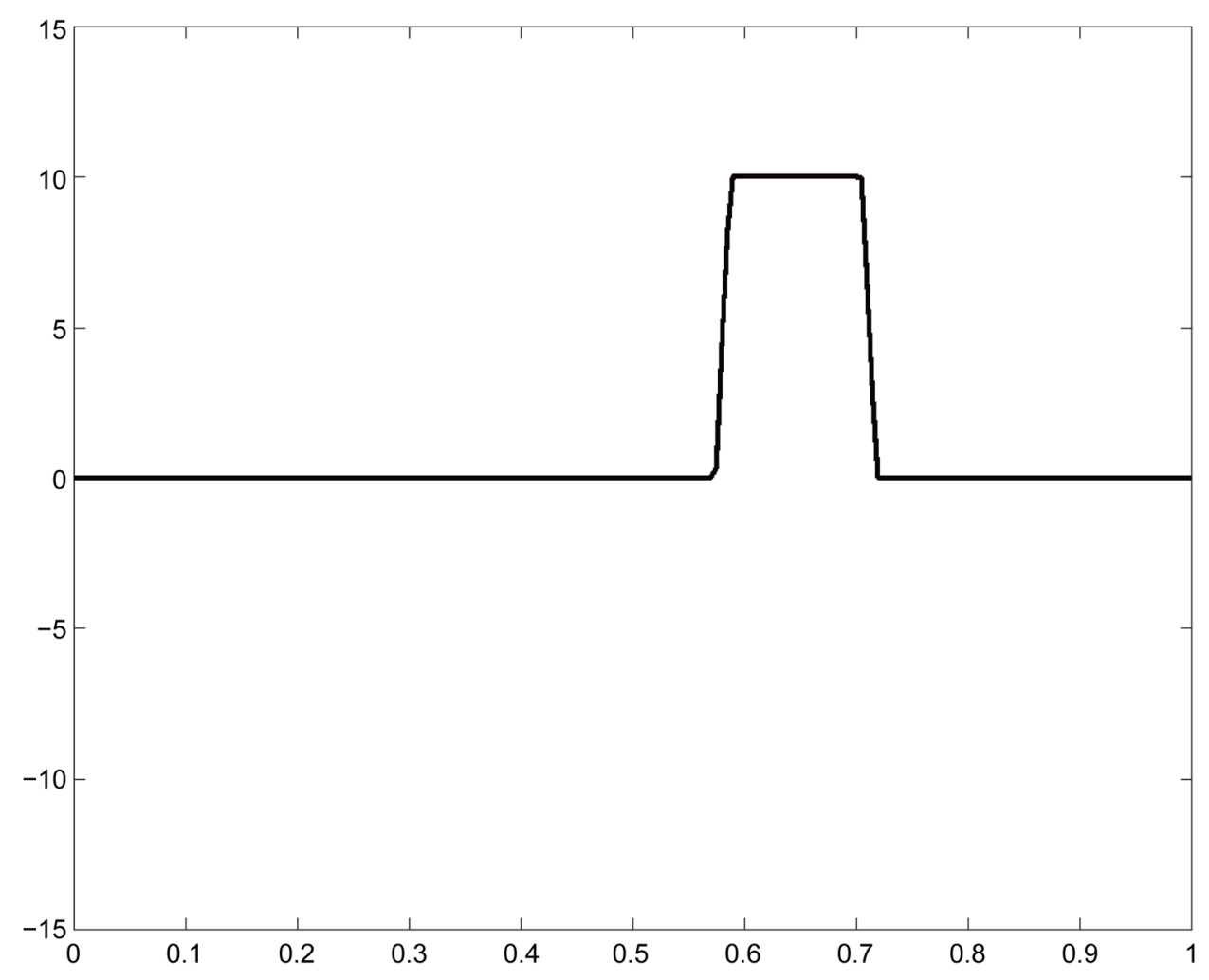

Figure 10. Optimal control with $\gamma=5$ and tracking objective given by (28). 


\section{Conclusion}

A framework for the optimal control of probability density functions of jump-diffusion processes was discussed. In this framework, two different, discrete-in-time and continuous-in-time, tracking functionals were considered together with a sparsity promoting $L^{1}$-cost of the control. The resulting nonsmooth minimization problems governed by a Fokker-Planck partial integro-differential equation were investigated. The existence of at least an optimal control solution was proven. To characterize and compute the optimal controls, the corresponding first-order optimality systems were derived and their numerical approximation was discussed. These optimality systems in combination with a proximal scheme allowed to formulate an efficient solution procedure, which was also theoretically discussed. Results of numerical experiments were presented to validate the computational effectiveness of the proposed method.

\section{Acknowledgements}

Supported by the European Union under Grant Agreement Nr. 304617 "Multi-ITN STRIKE-Novel Methods in Computational Finance". This publication was supported by the Open Access Publication Fund of the University of Würzburg. We thank very much the Referee for improving remarks.

\section{References}

[1] Cont, R. and Tankov, P. (2004) Financial Modeling with Jump Processes. Chapman \& Hall, London.

[2] Kou, S.G. (2002) A Jump-Diffusion Model for Option Pricing. Management Science, 48, 1086-1101. http://dx.doi.org/10.1287/mnsc.48.8.1086.166

[3] Merton, R.C. (1976) Option Pricing When Underlying Stock Returns Are Discontinuous. Journal of Financial Economics, 3, 125-144. http://dx.doi.org/10.1016/0304-405X(76)90022-2

[4] Pascucci, A. (2011) PDE and Martingale Methods in Option Pricing. Springer, Berlin. http://dx.doi.org/10.1007/978-88-470-1781-8

[5] Harrison, J.M. and Pliska, S.R. (1983) A Stochastic Calculus Model of Continuous Trading: Complete Markets. Stochastic Processes and Their Applications, 15, 313-316. http://dx.doi.org/10.1016/0304-4149(83)90038-8

[6] Fleming, W.H. and Soner, M. (1993) Controled Markov Processes and Viscosity Solutions. Springer, Berlin.

[7] Pham, H. (2009) Continuous-Time Stochastic Control and Optimization with Financial Applications. Springer, Berlin. http://dx.doi.org/10.1007/978-3-540-89500-8

[8] Yong, J. and Zhou, X.Y. (2000) Stochastic Controls, Hamiltonian Systems and Hamilton-Jacobi-Bellman Equations. Springer, Berlin.

[9] Annunziato, M. and Borzì, A. (2013) A Fokker-Planck Control Framework for Multimensional Stochastic Processes. Journal of Computational and Applied Mathematics, 237, 487507. http://dx.doi.org/10.1016/j.cam.2012.06.019

[10] Annunziato, M. and Borzì, A. (2010) Optimal Control of Probability Density Functions of Stochastic Processes. Mathematical Modelling and Analysis, 15, 393-407. http://dx.doi.org/10.3846/1392-6292.2010.15.393-407 
[11] Roy, S., Annunziato, M. and Borzì, A. (2016) A Fokker-Planck Feedback Control-Constrained Approach for Modelling Crowd Motion. Journal of Computational and Theoretical Transport, 442-458. http://dx.doi.org/10.1080/23324309.2016.1189435

[12] Cox, D.R. and Miller, H.D. (1977) The Theory of Stochastic Processes. CRC Press, Boca Raton.

[13] Schuss, Z. (2010) Theory and Applications of Stochastic Processes: An Analytical Approach. Springer, Berlin. http://dx.doi.org/10.1007/978-1-4419-1605-1

[14] Stroock, D.W. (2003) Markov Processes from K. Itô's Perspective. Princeton University Press, Princeton. http://dx.doi.org/10.1515/9781400835577

[15] Borzì, A. and Schulz, V. (2012) Computational Optimization of Systems Governed by Partial Differential Equations. SIAM, Philadelphia.

[16] Lions, J.L. (1971) Optimal Control of Systems Governed by Partial Differential Equations. Springer, Berlin.

[17] Tröltzsch, F. (2010) Optimal Control of Partial Differential Equations: Theory, Methods and Applications. American Mathematical Society. http://dx.doi.org/10.1090/gsm/112

[18] Borzì, A. and Gonzalez, S. (2015) Andrade, Second-Order Approximation and Fast Multigrid Solution of Parabolic Bilinear Optimization Problems. Advances in Computational Mathematics, 41, 457-488. http://dx.doi.org/10.1007/s10444-014-9369-9

[19] Schindele, A. and Borzì, A. (2016) Proximal Methods for Elliptic Optimal Control Problems with Sparsity Cost Functional. Applied Mathematics, 7, 967-992. http://dx.doi.org/10.4236/am.2016.79086

[20] Jäger, S. and Kostina, E.A. (2005) Parameter Estimation for Forward Kolmogorov Equation with Application to Nonlinear Exchange Rate Dynamics. PAMM, 5, 745-746. http://dx.doi.org/10.1002/pamm.200510347

[21] Borzì, A., Ito, K. and Kunisch, K. (2002) Optimal Control Formulation for Determining Optical Flow. SIAM Journal on Scientific Computing, 24, 818-847. http://dx.doi.org/10.1137/S1064827501386481

[22] Stadler, G. (2009) Elliptic Optimal Control Problems with $L^{1}$-Control Costs and Applications for the Placement of Control Devices. Computational Optimization and Applications, 44, 159-181. http://dx.doi.org/10.1007/s10589-007-9150-9

[23] Ulbrich, M. (2011) Semismooth Newton Methods for Variational Inequalities and Constrained Optimization Problems in Function Spaces. SIAM, Philadelphia. http://dx.doi.org/10.1137/1.9781611970692

[24] Nesterov, Y.E. (1983) A Method for Solving the Convex Programming Problem with Convergence Rate $\mathrm{O}\left(1 / \mathrm{k}^{2}\right)$. Soviet Mathematics Doklady, 27, 372-376.

[25] Tyrrell Rockafellar, R. (1976) Monotone Operators and the Proximal Point Algorithm. SIAM Journal on Control and Optimization, 14, 877-898.

http://dx.doi.org/10.1137/0314056

[26] Bauschke, H.H., Burachik, R.S., Combettes, P.L., Elser, V., Luke, D.R. and Wolkowicz, H. (2011) Fixed-Point Algorithms for Inverse Problems in Science and Engineering. Springer, Berlin. http://dx.doi.org/10.1007/978-1-4419-9569-8

[27] Combettes, P.L. and Wajs, V.R. (2005) Signal Recovery by Proximal Forward-Backward Splitting. Multiscale Modeling \& Simulation, 4, 1168-1200.

http://dx.doi.org/10.1137/050626090

[28] Ochs, P.Y. and Cooper, G. (2014) IPiano: Inertial Proximal Algorithm for Nonconvex Optimization. SIAM Journal on Imaging Sciences, 7, 1388-1419.

http://dx.doi.org/10.1137/130942954 
[29] Garroni, M.G. and Menaldi, J.L. (1992) Green Functions for Second-Order Parabolic Integro-Differential Problems. Longman, London.

[30] Addou, A. and Benbrik, A. (2002) Existence and Uniqueness of Optimal Control for a Distributed-Parameter Bilinear System. Journal of Dynamical Control Systems, 8, 141-152. http://dx.doi.org/10.1023/A:1015372725255

[31] Fursikov, A.V. (2000) Optimal Control of Distributed Systems. Theory and Applications. American Mathematical Society.

[32] Ekeland, I. and Témam, R. (1999) Convex Analysis and Variational Problems. SIAM, Philadelphia.

[33] Gaviraghi, B., Annunziato, M. and Borzì, A. (2015) Analysis of Splitting Methods for Solving a Partial-Integro Fokker-Planck Equation. Applied Mathematics and Computation, 294, 1-17. http://dx.doi.org/10.1016/j.amc.2016.08.050

[34] Schiesser, W.E. (1991) The Numerical Method of Lines. Academic Press, Pittsburgh.

[35] Chang, J.S. and Cooper, G. (1970) A Practical Difference Scheme for Fokker-Planck Equations. Journal of Computational Physics, 6, 1-16. http://dx.doi.org/10.1016/0021-9991(70)90001-X

[36] Mohammadi, M. and Borzì, A. (2015) Analysis of the Chang-Cooper Discretization Scheme for a Class of Fokker-Planck Equations. Journal of Numerical Mathematics, 23, 125-144. http://dx.doi.org/10.1515/jnma-2015-0018

[37] Strang, G. (1968) On the Construction and Comparison of Difference Schemes. SIAM Journal on Numerical Analysis, 5, 506-517. http://dx.doi.org/10.1137/0705041

[38] Marchuk, G.I. (1981) Methods of Numerical Mathematics. Springer, Berlin.

[39] Hundsdorfer, W. and Verwer, J.G. (2010) Numerical Solutions of Time-Dependent Advection-Diffusion-Reaction Equations. Springer, Berlin.

[40] Geiser, J. (2009) Decomposition Methods for Differential Equations: Theory and Applications. Chapman \& Hall, London. http://dx.doi.org/10.1201/9781439810972

[41] Schindele, A. and Borzì, A. (2016) Proximal Schemes for Parabolic Optimal Control Problems with Sparsity Promoting Cost Functionals. International Journal of Control.

\section{Submit or recommend next manuscript to SCIRP and we will provide best service for you:}

Accepting pre-submission inquiries through Email, Facebook, LinkedIn, Twitter, etc.

A wide selection of journals (inclusive of 9 subjects, more than 200 journals)

Providing 24-hour high-quality service

User-friendly online submission system

Fair and swift peer-review system

Efficient typesetting and proofreading procedure

Display of the result of downloads and visits, as well as the number of cited articles

Maximum dissemination of your research work

Submit your manuscript at: http://papersubmission.scirp.org/

Or contact am@scirp.org 